Ann. Geophysicae 16, 787-803 (1998) @ C EGS - Springer-Verlag 1998

\title{
ULF waves: 1997 IAGA division 3 reporter review
}

\author{
K. Takahashi \\ Solar-Terrestrial Environment Laboratory, Nagoya University, Toyokawa 442, Japan \\ Also at: The Johns Hopkins University Applied Physics Laboratory, Johns Hopkins Road, Laurel, MD 20723-6099, USA
}

Received: 25 November 1997 / Accepted: 12 December 1997

\begin{abstract}
Highlights of studies of ULF waves from 1995 to early 1997 are presented. The subjects covered include (1) Pc 3-5 waves excited by sources in the solar wind, with emphasis on the role of the magnetospheric cavity in modifying the external source and establishing its own resonances, and the role of the plasmapause in magnetohydrodynamic wave propagation; (2) Pi 2 waves, with emphasis on the plasmaspheric resonances and possible alternative excitation by plasmasheet source waves; (3) the spatial structure of internally excited long-period waves, including a kinetic theory for radially confined ring current instability and groundbased multipoint observation of giant pulsations; (4) amplitude-modulated Pc 1-2 waves in the outer magnetosphere (Pc 1-2 bursts) and in the inner magnetosphere (structured Pc 1 waves or pearls); and (5) the source region of the quasi-periodic emissions. Theory and observations are compared, and controversial issues are highlighted. In addition, some future directions are suggested.
\end{abstract}

Key words. Magnetospheric physics (MHD waves and instabilities; Plasma waves and instabilities; Solar wind magnetosphere interactions)

\section{Introduction}

This study is intended to give highlights of studies of ULF waves in the magnetosphere for the period from 1995 to mid-1997, preceding the 8th Assembly of the International Association of Geomagnetism and Aeronomy (IAGA). The subject area of ULF waves as defined in the description of the IAGA divisions includes the morphology, excitation, propagation, detection, and geophysical significance and effects of ULF waves.
This review is based on papers selected from over 150 publications in international geophysical journals since 1995. About two-thirds of these publications present observations or a combination of observations and theoretical models. The rest present theoretical studies including numerical simulations. Most of the pulsations discussed in the reports of experimental work can be classified according to the conventional $\mathrm{Pc} / \mathrm{Pi}$ scheme, but there are many basic theoretical studies that examine the general properties of magnetohydrodynamic (MHD) waves without reference to specific observed wave types. The overall trend in the past two years has been the increased use of numerical simulations and models and their comparison with real observations. In some cases, satellites were flown through the simulation box to give fairly realistic magnetic field observations. This approach was typical for the solar-wind-driven long-period pulsations. By comparison, there were fewer theoretical papers for long-period pulsations generated within the magnetosphere by kinetic processes. In the area of Pc 12 waves, however, there are a quite a few publications on the theory. An increased number of ground and satellite observations of ion cyclotron waves was the apparent reason for the elevated theoretical effort. Since the theory of Pc 1 waves will be covered by another review on wave-particle interaction, the present review of Pc 1 waves will focus on their observational aspects. Nonlinear effects (in particular, the ponderomotive wave forces), which is the subject of a number of theoretical papers (e.g., Guglielmi et al., 1995; Rankin et al., 1995; Feygin et al., 1997), will not be included. For a recent development in this area readers are referred to the review by Allan and Manuel (1996). Other aspects of low-frequency wave-particle interaction have been reviewed by Uberoi (1995). For a comprehensive summary of studies of ULF waves prior to 1994, see Engebretson (1995).

The organization of the work is as follows. Section 2 reviews a debate on the validity of the concept of fieldline resonance. Section 3 reviews studies of externally driven long-period waves. Section 4 reviews studies of 
internally excited long-period waves. Section 5 reviews studies of internally excited short-period waves. Section 6 presents conclusions.

\section{Is the field-line resonance concept valid?}

Because the single-fluid MHD equations are fundamental to studies of ULF waves, it is appropriate to begin this article by reviewing a debate concerning the concept of field-line resonance (FLR) derived from the equations. The validity of the FLR concept has been questioned by Bellan (1994, 1996), who uses two-fluid (which includes electron inertia) equations to describe wave propagation in the magnetosphere. Using the usual box magnetosphere with a plasma inhomogeneity in the $x$ direction, the wave equation for the azimuthal electric field $E_{y}$ can be cast in the form (see Wright and Allan, 1996a)

$A_{4} \frac{\mathrm{d}^{4} E_{y}}{\mathrm{~d} x^{4}}+A_{3} \frac{\mathrm{d}^{3} E_{y}}{\mathrm{~d} x^{3}}+A_{2} \frac{\mathrm{d}^{2} E_{y}}{\mathrm{~d} x^{2}}+A_{1} \frac{\mathrm{d} E_{y}}{\mathrm{~d} x}+A_{0} E_{y}=0$.

Bellan (1994) shows that the coefficient $A_{4}$ of the highest-order term does not vanish at the location $\left(x=x_{A}\right)$ where $\omega^{2}=k_{z}^{2} V_{A}^{2}$ is satisfied, which means that $x_{A}$ is not a singularity of the differential Eq. (1), unlike the case of the single-fluid wave equation (Southwood, 1974). Here $\omega$ is the wave frequency, $k_{z}$ is the wave number parallel to the ambient magnetic field, and $V_{A}$ is the Alfvén velocity. Taken literally, this would mean that resonant excitation of local shear Alfvén waves by external compressional waves cannot happen and that the concept of FLR needs to be totally revised.

Fortunately, this is not the case in practice, according to Wright and Allan (1996a). They argue that although Eq. (1) is mathematically valid it is not likely that the two-fluid equation becomes relevant to most ULF waves observed in the real magnetosphere. They point out that each wave equation has a domain of validity and that it is necessary to make a quantitative evaluation of relevant spatial and temporal scales to find out when an equation loses its validity and requires higher-order correction. Wright and Allan (1996a) show that the observed pulsations have lifetimes that are shorter than the time scale for the two-fluid effect to set in. The lifetime of a typical magnetic pulsation in the Pc $3-5$ band is 5 to 20 cycles. By comparison, about 2000 cycles are required before the two-fluid effect becomes significant. This means that under normal circumstances, ULF waves can be adequately described by the single-fluid equation, which can be obtained by setting $A_{4}=A_{3}=0$ in Eq. (1). Note that the singularity in the single-fluid equation is not a physical reality, either. The ionospheric dissipation or some other process will remove the singularity before the system becomes singular, and magnetic pulsations will always have a finite lifetime. The important point is that some properties of the pulsations, such as their polarization and latitudinal localization, can be adequately described by the single-fluid wave equation. In related work, Wright and Allan (1996b) show that the resistivity within the magnetospheric plasma and the finite Pedersen conductivity in the ionosphere lead to quite similar behavior of Alfvén waves. Thus, regardless of the dissipation mechanism, the traditional single-fluid MHD can give a fairly robust description of how field lines oscillate in a practical time scale for observation of ULF waves.

\section{Relative importance of externally applied periodic pressure changes and internally generated compressional eigenmodes}

Many studies of pulsations in the Pc 3-5 and Pi 2 bands (period $=10-600 \mathrm{~s})$ discuss the possible relation between their spectral properties and the cavity or wave guide eigenmodes. Various pulsations observed by satellites and ground-based experiments were subjected to careful analysis in the search for evidence of the cavity/waveguide mode predicted by theory. Our understanding of these pulsations has considerably improved compared to one decade ago (e.g., Yumoto, 1986).

\subsection{A brief review of the problem}

The modern concept of the magnetospheric cavity mode, which was introduced a decade ago (Kivelson et al., 1984), has been studied in great detail using analytical and numerical techniques. The theoretical model for the cavity mode started from a simple box geometry with perfectly reflecting boundaries (Kivelson and Southwood, 1986). More-recent studies use other magnetospheric models, including a cylindrical magnetosphere (Allan et al., 1986a) and dipole magnetosphere (Lee and Lysak, 1989). A variation of the box model is a rectangular wave guide (Samson et al., 1992). The differences in the magnetospheric geometry lead to different mode structures.

An important factor to be considered in addition to cavity geometry is loss of the fast-mode energy by various mechanisms. A hierarchy of magnetospheric models is illustrated in Fig. 1 with regard to energy loss. First, as described in the theory of field-line resonance (Southwood, 1974), fast-mode energy can be lost through mode conversion into the shear Alfvén waves. Also, if a finite ionospheric conductivity is taken into account, Joule dissipation becomes a significant loss mechanism (Allan and Poulter, 1989; Wright and Allan, 1996b). The introduction of the wave guide geometry allows fast-mode energy to escape tailward while the waves are reflected at radial boundaries, establishing a radial eigenmode structure. Finally, it is possible for fast-mode energy to be lost into the solar wind through the magnetopause (Fujita and Glassmeier, 1995; Lee, 1996). Obviously, loss of fast-mode energy through these mechanisms reduces the $Q$ value of the magnetospheric cavity mode. Whether one can detect the compressional eigenmodes as narrow-band oscillations should depend critically on how quickly the fast-mode 
Fast-mode energy loss mechanisms

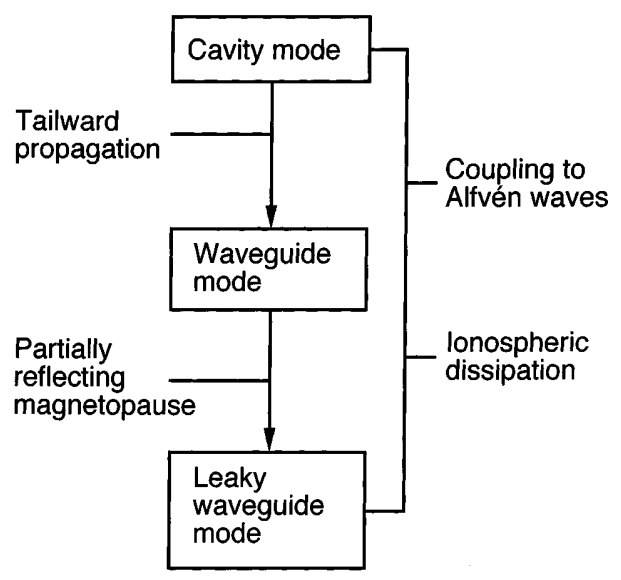

Fig. 1. Magnetospheric models used to study ULF wave propagation. Various models with different geometries and energy loss mechanisms have been used in theoretical studies

energy is lost out of the cavity. No numerical studies have made a comprehensive assessment of the energyloss mechanisms, so the debate continues about whether the cavity mode can be experimentally detected.

Another facor that must be considered in trying to identify the magnetospheric cavity/waveguide mode is the type of solar wind variations that impinge on the magnetopause. As illustrated in Fig. 2 (top), a multiharmonic cavity mode may be excited if the solar wind

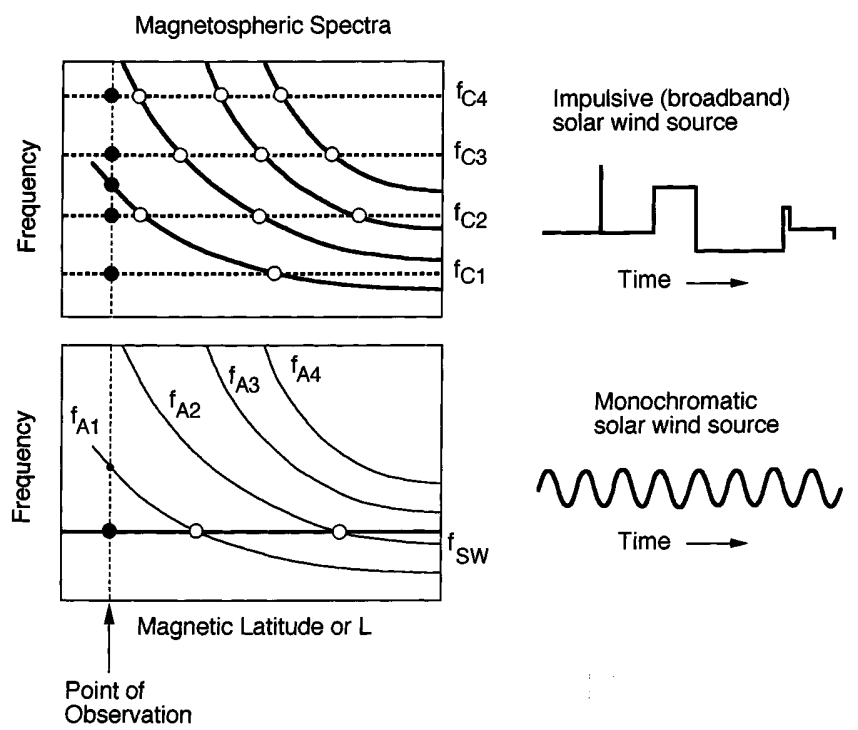

Fig. 2. Illustrations of two types of ULF sources in the solar wind and the magnetospheric response. In the case illustrated at the top, the source consists of irregular and impulsive variations. This is effectively a broadband source, which can excite a number of cavity mode oscillations and multiharmonic standing Alfvén waves (Alfvén continuum) in the magnetosphere. In the case illustrated at the bottom, the solar wind source is a sinusoid. This source causes the magnetosphere to oscillate at the same frequency with little trace of the Alfvén continuum except on $L$ shells on which the Alfvén frequency matches the source frequency. The size of the solid circles indicates the wave intensity at an observation point pressure pulses randomly hit the magnetopause, since such a time series provides broadband stimuli to magnetospheric fast-mode waves. According to computer simulations assuming perfectly reflecting boundaries (e.g., Lee and Lysak, 1991a), not only the cavity mode but also the multiharmonic toroidal resonances (Alfvén continuum) are excited by the impulsive source. In contrast, when the solar wind pressure (or some other external quantity) changes periodically in a sinusoidal manner, the magnetospheric field will oscillate with the same periodicity as illustrated in Fig. 2 (bottom). According to numerical simulations (Lee and Lysak, 1991b), the compressional oscillations can couple to toroidal-mode standing Alfvén waves at the locations where the driver frequency matches the local toroidalmode Alfvén frequency. The compressional wave in this case mimics the behavior of the global cavity mode/wave guide modes, but its origin is not the fast-mode waves trapped in the magnetosphere. Distinguishing between the forced oscillations and the standing compressional oscillations of the magnetospheric cavity requires a careful inspection of the solar wind condition and the mode structure of magnetic field perturbations in the magnetosphere.

In the following subsections we review the source/ resonator problem for a few representative wave types.

\subsection{Pc 5 waves}

The proposition by Harrold and Samson (1992) and Samson et al. (1992) that pulsations with discrete frequencies of 1.3, 1.9, 2.6, 3.4, and $4.2 \mathrm{mHz}$ observed by high-latitude radar (Samson et al., 1991; Walker et al., 1992) arise from a wave guide mode on the flankside of the magnetosphere motivated several studies that searched for pulsations having these frequencies. (This proposition is referred to as the cavity mode model of Samson et al., CMS, by Ziesolleck and McDiarmid, 1995). In the model, the wave guide mode is produced by wave reflection at a turning point $x_{t}$ in the magnetosphere and at an outer boundary $x_{m}$. The turning point for a wave having frequency $\omega$ and wave vector $\mathbf{k}$ is given by

$\omega^{2}-V_{A}^{2}\left(x_{t}\right)\left(k_{y}^{2}\left(x_{t}\right)+k_{z}^{2}\left(x_{t}\right)\right)=0$.

The eigenfrequencies $\omega_{n, l}$ of the wave guide mode are given by

$$
\int_{x_{t}}^{x_{m}}\left(\frac{\omega_{n, l}}{V_{A}^{2}}-k_{y}^{2}(x)-k_{z, l}^{2}(x)\right)^{1 / 2} \mathrm{~d} x-(n-\alpha) \pi=0
$$

where $k_{z, l}(\mathrm{x})$ are the field-aligned wave numbers and $\alpha$ is a phase factor depending on the reflection conditions. The parameters $n$ and $l$ specify the radial and fieldaligned mode numbers, respectively. Samson et al. (1992) and Walker et al. (1992) report that CMS frequencies vary little and do not depend on the geomagnetic condition. 
There are studies in support of the presence of CMS frequencies at different latitudes and from different instruments. Shimazu et al. (1995) report multipoint observations of a Pc 5 pulsation having discrete frequencies of $3.3,4.7,5.9$, and $7.1 \mathrm{mHz}$ following a minor increase in the solar wind dynamic pressure, at latitudes ranging from the auroral zone to the geomagnetic equator. Provan and Yeoman (1997) use the Wick radars $(L=4-6.5)$ and find some of the CMS frequencies at midlatitude. An additional, and surprising, report of the CMS frequencies comes from magnetometer observations at a low latitude $(L=1.6)$ station. Francia and Villante (1997) show that spectra averaged over many hours exhibit peaks at or near the CMS frequencies (Fig. 3) and that they are seen even on the nightside.

Some studies, in contrast, indicate that pulsations can be observed at a variety of frequencies, and the authors question the stability of the CMS frequencies. Ziesolleck and McDiarmid (1995) present a critical examination of the CMS frequencies based on the CANOPUS magnetometer data covering $L=4.2-12.3$. Although they confirm the signature of multiple discrete field-line resonances, they do not find the pulsation frequencies to be as stable as claimed from radar observations (Samson et al., 1991; Walker et al., 1992), except perhaps for the $1.9 \mathrm{mHz}$ component. The interpretation of this result is that either there are other mechanisms contributing to the high-latitude magnetic pulsations or

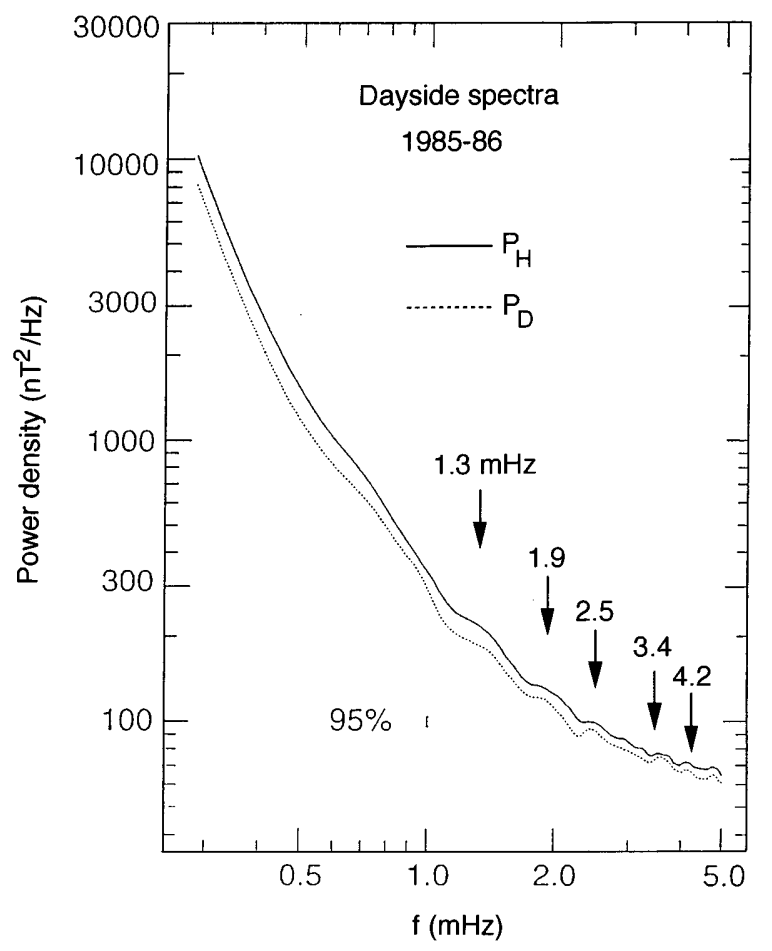

Fig. 3. An example of average power spectra for the horizontal $(\mathrm{H}$ and D) components of the magnetic field measured at a low-latitude ( $L=1.6$ ) ground station (adapted from Fig. 1a of Francia and Villante, 1997). The arrows indicate the discrete frequencies observed by high-latitude radar and attributed to wave guides modes the wave guide-mode frequencies vary with magnetospheric conditions. Shimazu et al. (1995) also note that of 9 Pc 5 events observed simultaneously at Kiruna (geographic latitude, $68^{\circ}$; longitude, $20^{\circ}$ ) and College (geographic latitude, $65^{\circ}$; longitude, $212^{\circ}$ ), the majority ( 8 events) had different frequencies at the longitudinally separated stations. Such cases can be attributed to pulsations excited locally, and it should not come as a surprise to find frequencies that do not match the CMS frequencies.

Finally, we note that there is a class of Pc 5 (and other) pulsations that are directly driven by periodic disturbances in the solar wind in the manner illustrated in Fig. 2 (bottom). Korotova and Sibeck (1995) and Matsuoka et al. (1995) use ground and geosynchronous (or near-geosynchronous) satellite observations along with a solar wind monitor and report Pc 5-band pulsations that are associated with similar periodic changes in the dynamic pressure of the solar wind. Matsuoka et al. (1995) demonstrate that pulsations seen on the ground can be explained by a small change in the Chapman-Ferraro current on the magnetopause induced by solar wind pressure variations. A similar but more persistent solar-wind-driven pulsation in the tail lobe is reported by Sarafopoulos (1995) using IMP-8 observations (Fig. 4 bottom panel). Because of the simultaneous solar wind measurements of the dynamic pressure by the ISEE 3 spacecraft (upper three panels), there is little doubt that the lobe oscillation is a direct

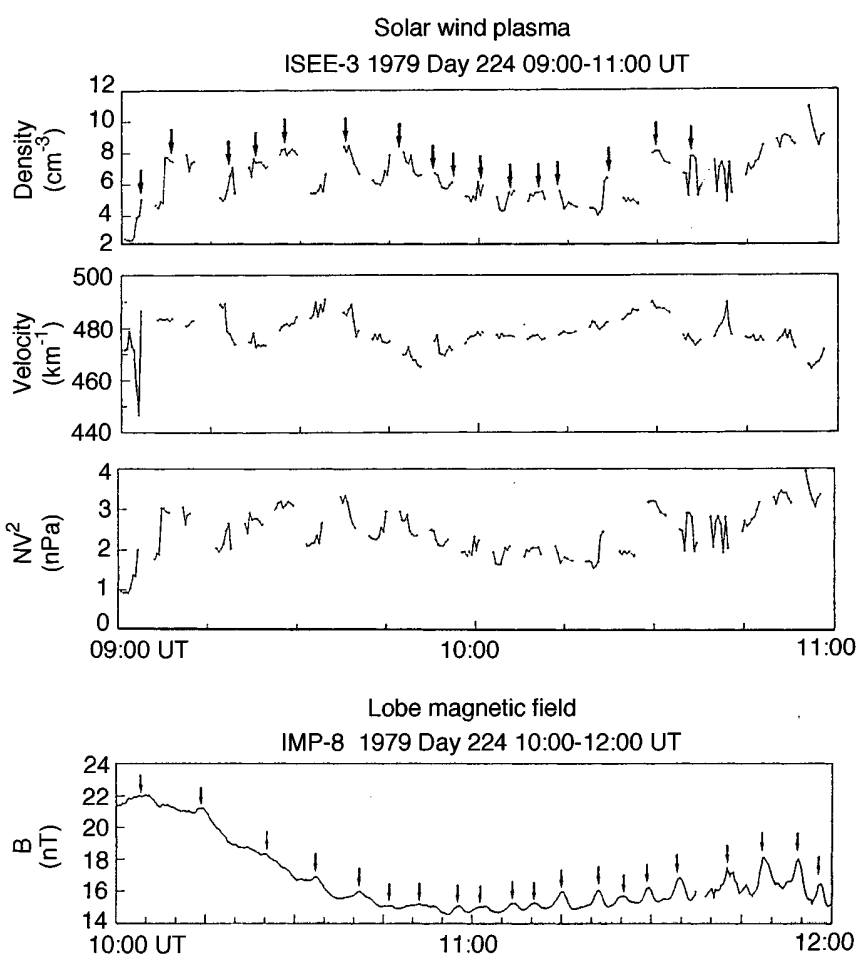

Fig. 4. An example of nearly identical Pc 5 pulsations observed in the solar wind plasma bulk parameters by ISEE 3 (top) and in the magnetotail magnetic field by IMP-8 (bottom) (adapted from Fig. 13 of Sarafopoulos, 1995). The solar wind and tail data are shifted by $1 \mathrm{~h}$ from each other to incorporate the solar wind flow from the ISEE 3 position (about $240 R_{\mathrm{E}}$ upstream) to the magnetosphere 
response to the solar wind oscillation. The Sarafopoulos event lasted $2-3 \mathrm{~h}$ and had a frequency of $1.7 \mathrm{mHz}$, which is quite close to one of the CMS frequencies. The pulsation reported by Korotova and Sibeck (1995) had a frequency of $2.2 \mathrm{mHz}$, and those reported by Matsuoka et al. (1995) also had a median frequency of $2.2 \mathrm{mHz}$.

From these examples it is clear that disturbances in the solar wind can be a direct cause of low-frequency pulsations observed on the ground and that wave guide modes are not necessarily the only cause of Pc 5 pulsations. The question is how often these solar-windinduced magnetic pulsations contribute to the pulsations observed on the ground by radar or magnetometers. Most statistical studies of $\mathrm{mHz}$-range pulsations on the ground (e.g., Ziesolleck and McDiarmid, 1995) have not fully incorporated solar wind data. It is of great importance to examine the state of the solar wind at times when magnetic pulsations are observed on the ground.

Not only does solar wind directly drive magnetospheric pulsations, but it should also control the frequency of magnetospheric resonances. As for the wave guide mode, we note that the distance of the outer boundary $x_{m}$ appearing Eq. (3), will become smaller for a larger solar wind dynamic pressure or a larger southward component of the interplanetary magnetic field (Sibeck et al., 1991). This variation in $x_{m}$, along with the changes in $x_{t}$ and $V_{A}$, should lead to a strong dependence of the cavity mode frequencies on solar wind condition. Once again, we emphasize the importance of combined ground and solar wind observations to test the wave guide model.

Also, it is important to examine satellite data to test the models for Pc 5 waves. Satellites frequently observe toroidal-mode oscillations (Anderson et al., 1990; Nosé et al., 1995; Potemra and Blomberg, 1996) in a wide range of $L$. A statistical examination of whether these toroidal modes attain a large amplitude at the CMS frequencies in the manner illustrated in Fig. 2 (top) will be a better test of the cavity/wave guide model than those based only on ground magnetometers.

\subsection{Pc 3-4 waves}

Magnetospheric cavity/wave guide-mode has been suggested to be responsible for low-latitude Pc 3 pulsations as well. Samson et al. (1995) estimate that the frequency separation of multiharmonic wave guide modes can be $3-5 \mathrm{mHz}$ and propose that the magnetosphere is filled with many of these harmonics, as illustrated in the upper part of Fig. 5. Standing Alfvén waves on local field lines can be excited by coupling to the waveguide modes when the frequencies of the two modes match. If the frequency separation of the wave guide mode is small, the resonant Alfvén waves are excited on $L$ shells separated by short distances. Because a magnetometer responds to ionospheric currents flowing not only just above the magnetometer but over a range of latitudes, the ionospheric currents associated with the Alfvén waves produce in the magnetometer power spectral data
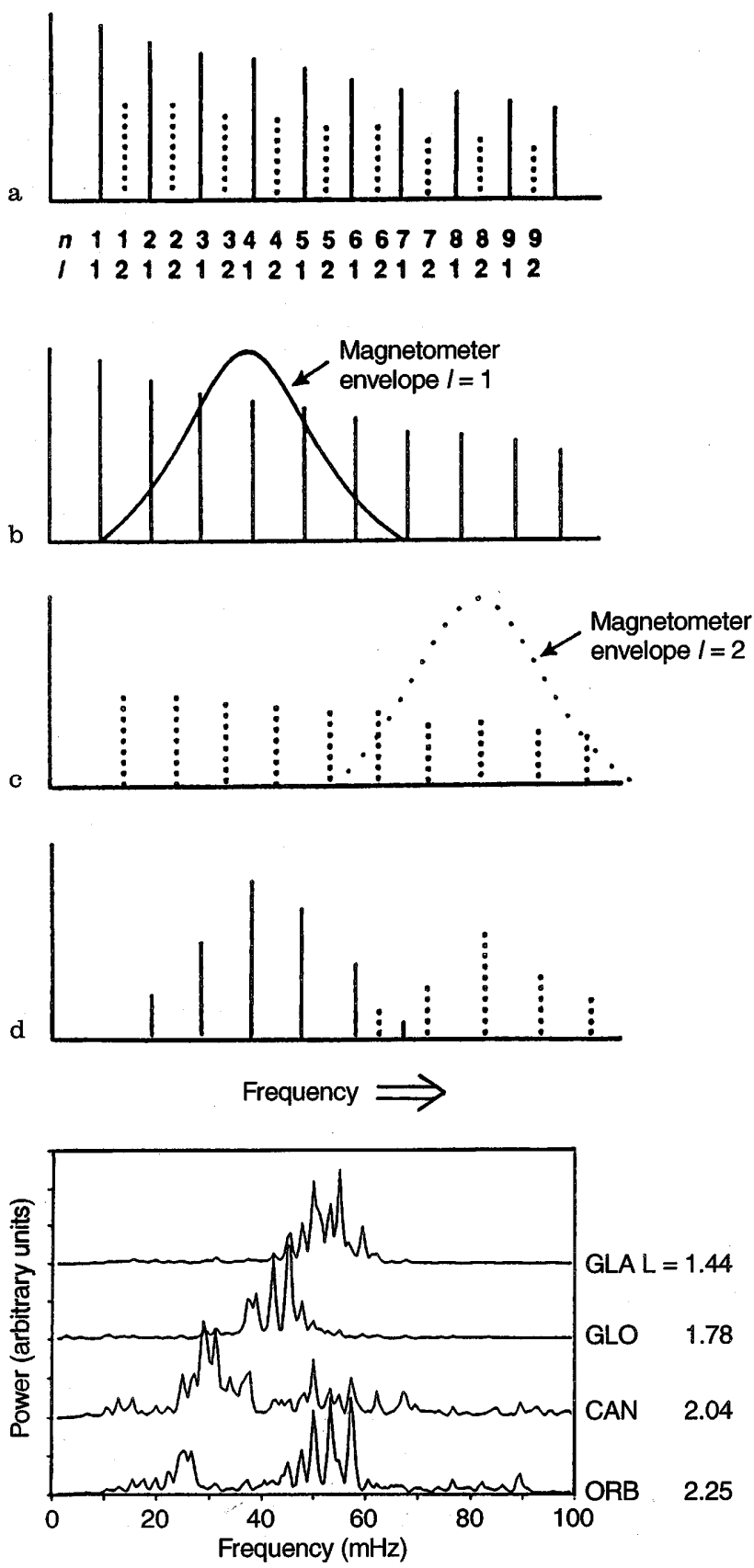

Fig. 5a-d. Model (top) and supporting low-latitude observations (bottom) of multiharmonic wave guide modes excited in the magnetosphere (adapted from Fig. 2 and 3 of Samson et al. 1995). In the model, wave guide modes are excited at frequencies $\omega_{n l}$, defined by Eq. (3) as illustrated in a. A magnetometer on the ground observes a spectrum that consists of multiple envelopes centered at the frequencies of the local standing Alfvén waves, as shown in $\mathbf{b}, \mathbf{c}$, and $\mathbf{d}$

a multiharmonic envelope structure that is centered at the frequencies of the harmonics of the local standing Alfvén wave. The fine structures of magnetic pulsations observed at four low-latitude ( $L=1.44-2.25)$ stations, shown in the lower part of Fig. 5, are attributed to this wave guide mechanism. Because the frequency of the standing Alfvén waves changes with $L$, the envelope structure associated with a given harmonic of the standing Alfvén wave also changes with $L$, as is clearly 
shown in this figure and verified using the cross-phase technique (Waters et al., 1995).

The frequency of (the envelope of) low-latitude pulsations does not always vary with $L$. There are observations in which Pc 3 pulsations exhibit a constant frequency over a range of latitudes. Feng et al. (1995) and Matsuoka et al. (1997) observe single-frequency Pc 3 events at low latitude $(L<2)$ using latitudinal arrays of magnetometers. Figure 6 shows an event represented by Feng et al. (1995). The pulsation was observed at four stations located from $L=1.17$ to 1.98 , and the most dominant oscillation had an identical frequency $(24 \mathrm{mHz})$ at all stations. Feng et al. (1995) estimate the $L$ dependence of the frequency of the fundamental-mode standing Alfvén wave (indicated by the solid curve) and find that the observed frequency matches the Alfvén frequency at $L \sim 2$. Consequently, they suggest that the observed single-frequency oscillations below $L=2$ are forced (i.e., nonresonant) oscillations associated with field-line resonance at $L \sim 2$ and that the possible driving source for the pulsations is the cavity mode oscillation trapped in the plasmasphere.

The idea of plasmaspheric cavity mode was proposed a long time ago by Saito and Matsushita (1968) for nightside Pi 2 pulsations, but it could be applied to

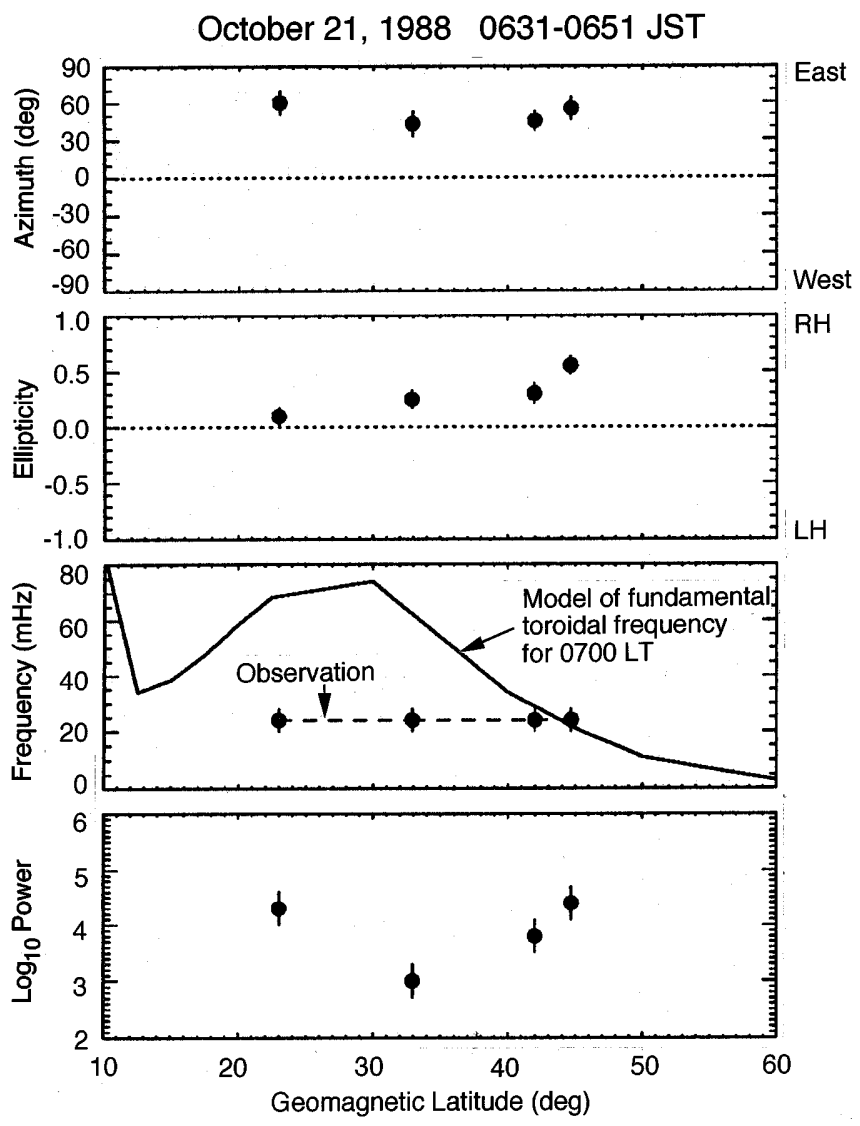

Fig. 6. Latitudinal profile of parameters characterizing a singlefrequency Pc 3 pulsation observed on the ground (adapted from Fig. 11 of Feng et al., 1995). The solid curve in the third panel indicates the frequency of fundamental-mode toroidal oscillation derived from a model dayside pulsations as well. When there is a sharp inward density gradient at the plasmapause, a cavity mode localized within the plasmasphere can be established. Quantitative analyses of the mode have been presented using a box magnetosphere (Zhu and Kivelson, 1989), a cylindrical magnetosphere (Allan et al., 1986b) and, most recently, a dipole magnetosphere (Fujita and Glassmeier, 1995; Lee, 1996). The simulation by Lee (1996), shown in Fig. 7, indicates that in addition to the multiharmonic global cavity mode that extends from the inner to the outer magnetosphere, there is a compressional mode having a frequency of $42 \mathrm{mHz}$ and localized between $L=3$ (the inner boundary of the simulation domain) and $L=5$ (the plasmapause). An approximate frequency $f$ of this plasmaspheric mode is related to the fast-mode bounce time between the inner and outer reflection (or turning) points $x_{1}$ and $x_{2}$, as in

$f=\left[2 \int_{x_{1}}^{x_{2}} \frac{\mathrm{d} x}{V_{A}}\right]^{-1}$

where the fast-mode velocity is approximated by the Alfvén velocity $V_{A}$ assuming a cold plasma. Fujita and Glassmeier (1995) use a cavity with a partially reflecting magnetopause and still get a well-defined plasmaspheric cavity mode. It appears that the plasmaspheric mode is a robust feature in simulations. The plasmaspheric cavity mode could be the source of the single-frequency oscillation observed at low latitude. However, this explanation may not hold in general.

One major problem with the cavity mode scenario for the low-latitude Pc 3 waves is the presence of a spectral component that is related to the interplanetary magnetic field (IMF) intensity through the well-known empirical relation

$f(\mathrm{mHz}) \sim 6 B_{\mathrm{IMF}}(\mathrm{nT})$.

This relation dates back to the pioneering work of Troitskaya et al. (1971) and has been supported by many statistical studies of Pc 3 waves and the solar wind. Most recently, Vellante et al. (1996) find in their 11-year statistical analysis of L'Aquila $(L \sim 1.6)$ magnetometer data a striking coexistence of two frequency components and the dependence of the frequencies on the solar cycle, as shown in Fig. 8. The higher-frequency component, according to a model calculation incorporating ionospheric mass loading, is consistent with local standing Alfvén waves. Meanwhile, the frequency of the lower-frequency group agrees with that given by relation (5) for long-term averages of the measured $B_{\mathrm{IMF}}$. The latter result can be explained by propagation of upstream waves into the magnetosphere and to the ground, as discussed later.

It is difficult to invoke the cavity/waveguide mode here because there is no obvious reason why the cavity mode frequencies should be controlled by the interplanetary magnetic field as in relation (5). Rather, the frequency should be determined by the cavity geometry and the mass distribution in it, and thus the frequency must be independent of the upstream wave frequency. 

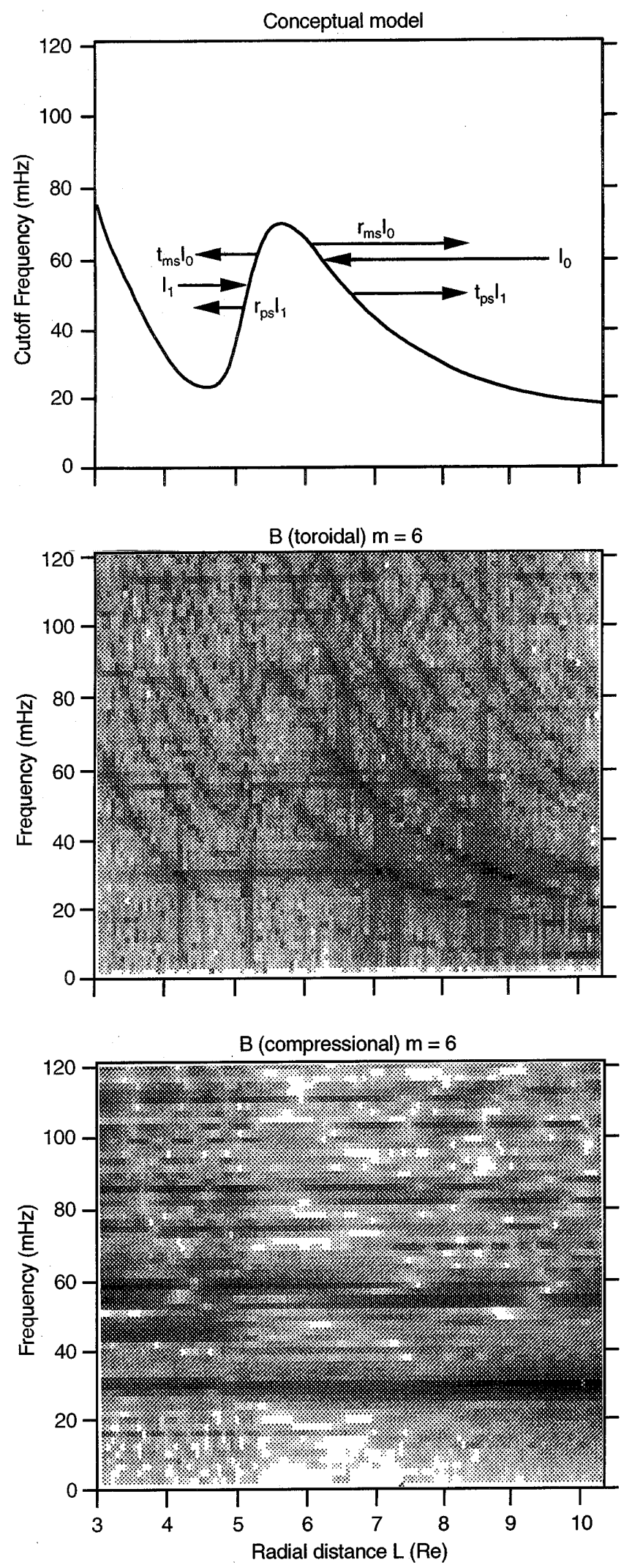

Fig. 7. Conceptual model (top) and the simulation results (middle and bottom) of magnetospheric ULF response to externally applied stimuli (adapted from Fig. 7 and 8 of Lee, 1996)

Note that for the case of the plasmaspheric cavity mode, the reflection (turning) point $x_{1}$ is a function of

frequency and wavenumber of the seed disturbance that enters the plasmasphere (Lee, 1996). This means that the cavity mode frequency also depends on the spatial and temporal structure of disturbances in the solar wind. However, it is still difficult to explain why the cavity mode frequency should vary as relation (5) predicts.

An explanation of single-frequency low-latitude Pc 3 pulsations which is consistent with the Vellante et al. (1996) result has been offered by Matsuoka et al. (1997). Instead of introducing the cavity resonance, Matsuoka et al. (1997) attribute the in-phase oscillations observed at $L<2.8$ to an evanescent mode associated with upstream waves that propagate into the deep magnetosphere. In their model, the waves hit a turning point located somewhere above $L=2.8$, and below this $L$ shell, the waves become evanescent and the magnetic field at different latitudes oscillates in phase. The frequency of the oscillation is the same as that of the upstream wave, which is consistent with the observation by Vellante et al. (1996).

The controversies over the cavity mode prompted Anderson and Engebretson (1995) to examine compressional-toroidal mode coupling in the Pc 3-4 band using magnetic field data acquired by the AMPTE CCE satellite. Previous spectral analyses of the same data set had indicated that discrete-frequency compressional pulsations are not evident in the satellite data (Engebretson et al., 1986). In the new study, Anderson and Engebretson (1995) confirm the absence of discrete compressional oscillations, but they also find high positive correlation between the amplitudes of the toroidal and compressional components. This implies that both components get energy from a common source and that coupling between the two modes occurs. Their interpretation of the origin of the compressional mode is as follows.

1. If the magnetosphere is a high- $Q$ cavity, the broadband compressional mode can be attributed to numerous compressional eigenmodes simultaneously excited in the magnetosphere (e.g., Samson et al., 1995).

2. If the magnetosphere is a low- $Q$ cavity, the broadband compressional waves represent energy continuously supplied from the solar wind and they should be considered to be propagating waves (i.e., Matsuoka et al., 1997).

As for observations in space, there may be an intrinsic difficulty in detecting the wave guide mode. A numerical simulation with a virtual spacecraft, performed by Rickard and Wright (1995), indicates that it may not be a simple task to detect the wave guide mode in satellite data. They find that the magnetometer signature of the fast mode in a wave guide, unlike a cavity, does not have a regular oscillatory nature with constant period over a range of $L$ shells. The comparison of the real satellite data (Lin et al., 1992) and simulated satellite observations, shown in Fig. 9, suggests that this kind of approach is quite useful in interpreting the data and in explaining why discrete-frequency oscillations are rarely seen in the magnetosphere. The physical reason for the weak compressional oscillation is that fast-mode waves are evanescent, some wave components propagate away 


\section{D-comp.}
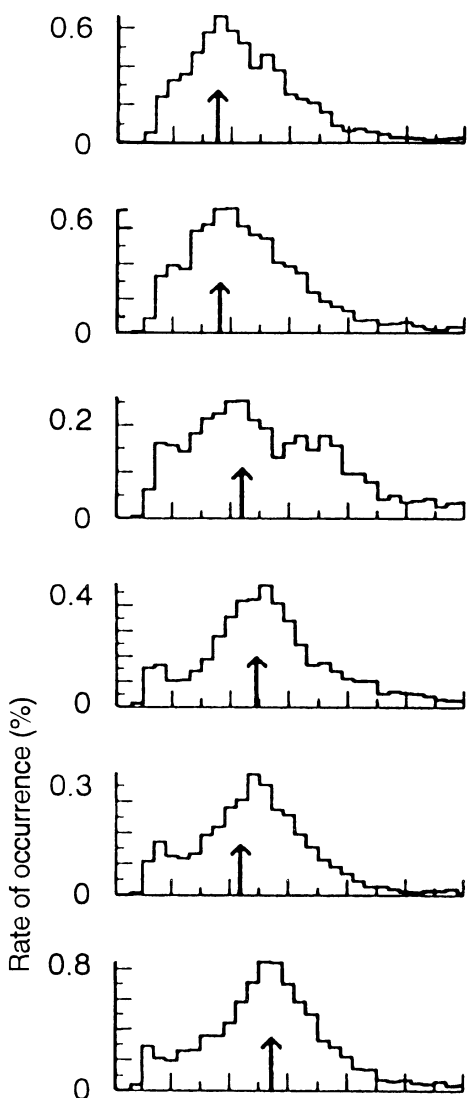

0.4
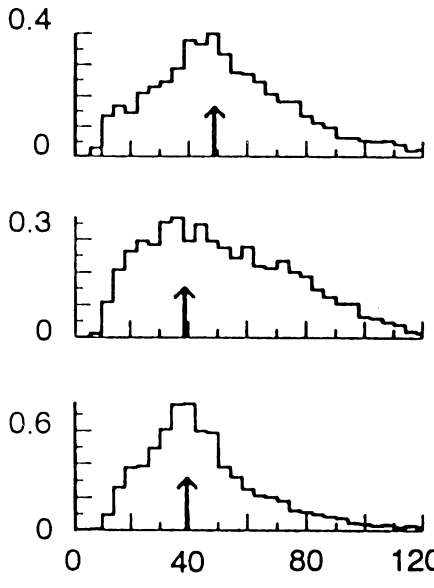

Frequency $(\mathrm{mHz})$

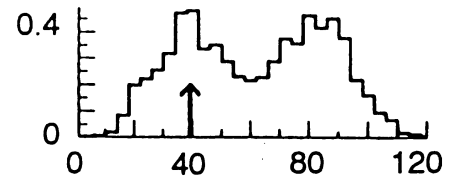

0.5
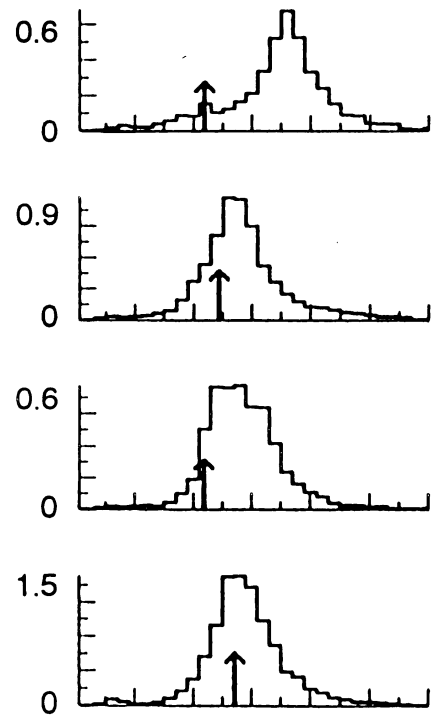

0.6

0
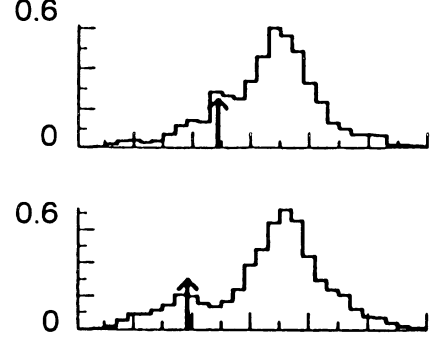

1985

$\overline{\mathrm{R}}=18$

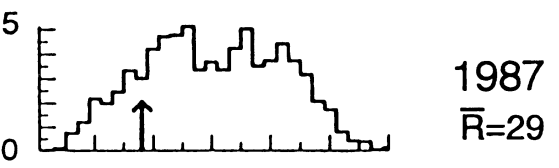

1988

$\overline{\mathrm{R}}=100$

1989

$\overline{\mathrm{R}}=158$

1990

$\bar{R}=142$.

1991

$\bar{R}=146$

1992

$\overline{\mathrm{R}}=94$

1993

$\overline{\mathrm{R}}=55$

Fig. 8. Frequency distribution of the peaks in the power spectra of the $\mathrm{H}$ and $\mathrm{D}$ component magnetic field records acquired at a low-latitude

1994 $(L=1.6)$ ground station (adapted from Fig. 1 of Vellante et al., 1996). The arrows indicate the frequency given by the empirical relation $f$ $(\mathrm{mHz})=6 B_{\mathrm{IMF}}(\mathrm{nT})$, where $B_{\mathrm{IMF}}$ is the annual average derived from the measurements in the solar wind azimuthally, and coupling with the shear Alfvén mode dampens waves with a small azimuthal wave number.

Perhaps the best way to detect the cavity mode in the magnetosphere is to use multisatellite observations. By measuring the coherence and phase delay of compressional pulsations between radially separated points, the relative importance of propagating and standing waves could be quantitatively determined.

\subsection{Pi 2 waves}

Pi 2 waves are also a phenomenon in which the cavity mode may play an important role. For the case of this nightside phenomenon, the absence of the magnetopause boundary and low reflection coefficient of the ionosphere warrant a different approach from that used with the dayside Pc 3-5 waves.

There is evidence that Pi 2 waves, when observed in the inner $(L<5)$ magnetosphere, exhibit properties of a radially standing compressional wave: an amplitude minimum and an associated $180^{\circ}$ phase reversal for the compressional magnetic field component (Takahashi et al., 1995). This observation is related to the earlier finding of the H-component phase reversal near $L=4$ reported by Björnsson et al. (1971). Whether there is a node of a standing wave at $L=4$ is not always clear, but there are observations indicating that the low- 

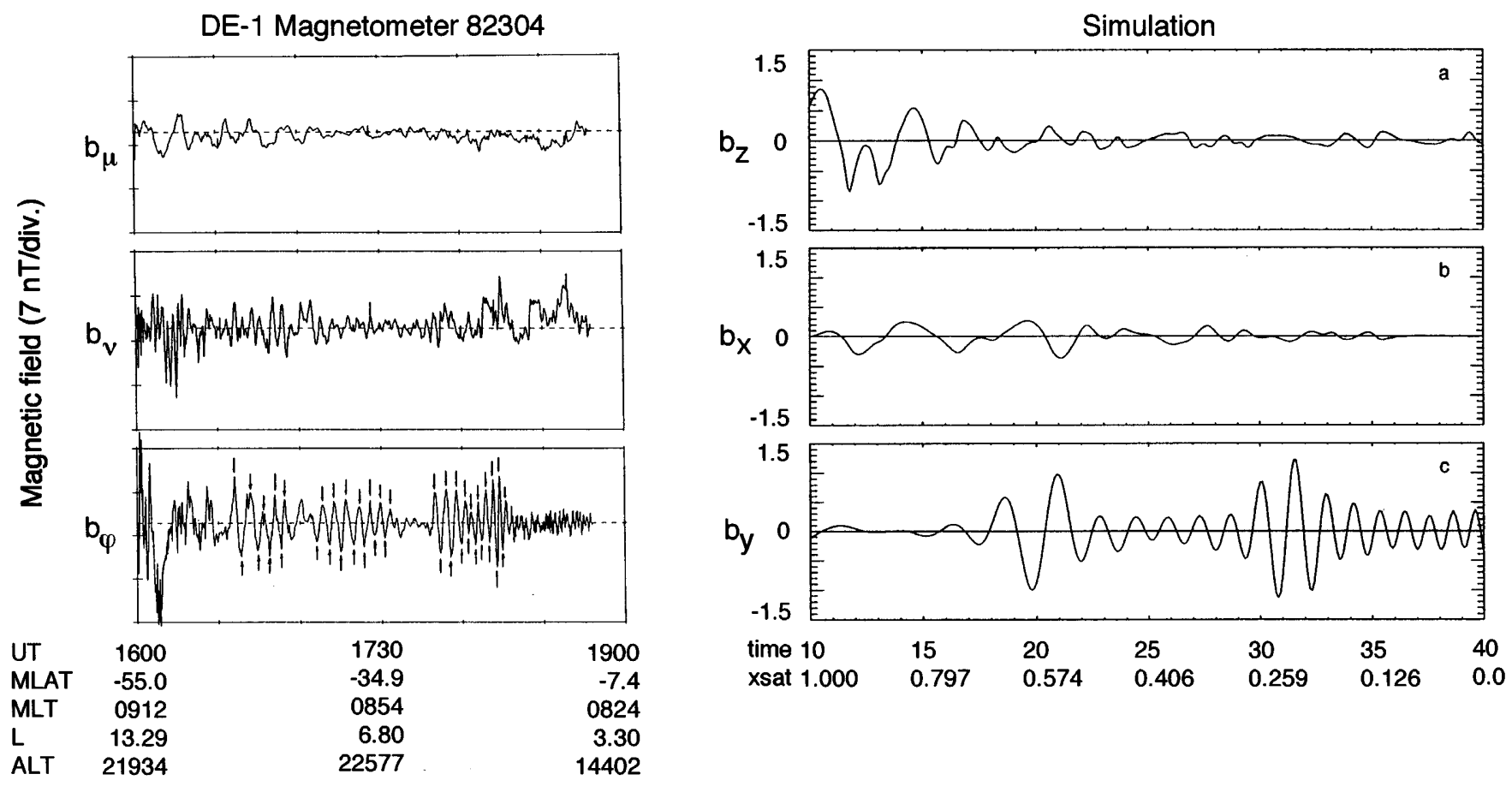

Fig. 9. Comparison of magnetic field time series from a satellite (left, adapted from Fig. 3b of Lin et al., 1992) and a wave guide simulation (right, adapted from Fig. 9 of Rickard and Wright, 1995). The field components from top to bottom are field-aligned, radial, and azimuthal

latitude Pi 2 pulsations do not extend much beyond $L \sim 5$. Figure 10, an example taken from Bradshaw and Lester (1997), shows that the period of Pi 2 pulsations at $62^{\circ}$ latitude detected by a magnetometer is shorter than that observed by radar at $64^{\circ}$, in support of an early radar study (Yeoman and Orr, 1989). From these observations, it is often suggested that the source of
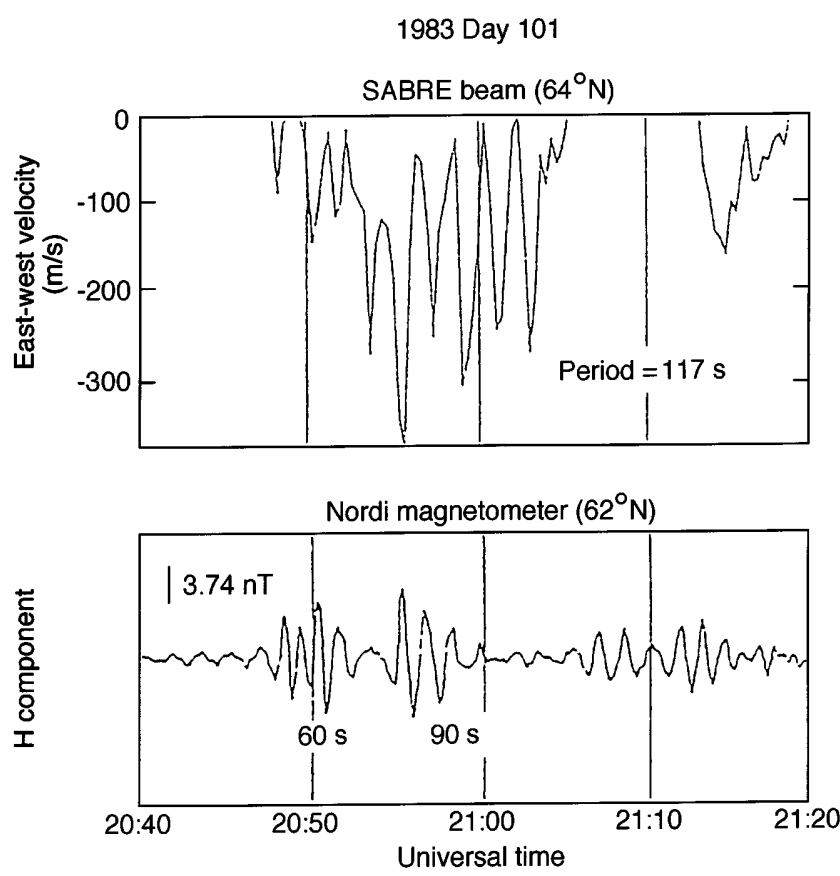

Fig. 10. Simultaneous observations of substorm-associated pulsations by the SABRE radar (top) and a ground magnetometer (bottom) (adapted from Fig. 6 of Bradshaw and Lester, 1997) low- and midlatitude $\mathrm{Pi} 2$ pulsations resides in the plasmasphere.

As discussed in Sect. 3.1, the real magnetosphere does not have perfectly reflecting boundaries, which is true in the nightside magnetosphere where fast-mode energy could easily escape into the magnetotail unless there is a strong mass density gradient, as at the inner edge of the plasma sheet. The influence of an open or closed outer boundary on Pi 2 wave-form and spatial phase structure was studied by Itonaga et al. (1997), and their results are schematically summarized in Fig. 11. They show that even when there is no outer reflecting boundary, a plausible situation in the nightside magnetosphere, a step-like disturbance incident on the plasmasphere still produces a Pi 2-type damped oscillation. However, in this case the relative phase between the plasmasphere and the plasmatrough is not 0 or $180^{\circ}$ but is near $90^{\circ}$, which contradicts satellite observations (i.e., Takahashi et al., 1995). In order to produce a 0 or $180^{\circ}$ phase delay, Itonaga et al. (1997) show by another model calculation that there must be a region of strong density gradient which forms an outer reflecting boundary, or alternatively, that the pulsations must be driven by an oscillating source.

Although the observations referenced above favor a Pi 2 source located in the plasmasphere, the difference in spatial phase structure between the model plasmaspheric response and satellite observations, as pointed out by Itonaga et al. (1997), suggests that we look for distant sources that might force plasmaspheric field lines to oscillate at a $\mathrm{Pi} 2$ frequency. Is there any observational evidence for such a source? Bauer et al. (1995) suggest that the Pi 2 source may be present at the inner edge of the plasmasheet. In AMPTE/IRM plasma and magnetic 


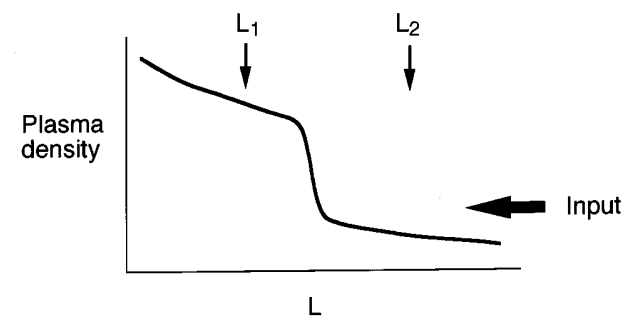

Step function Periodic forcing
Input time series

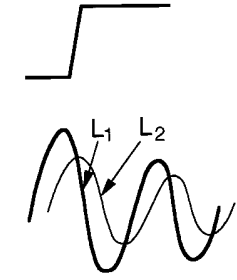

Response time series

Spatial phase delay

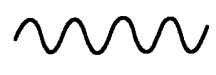

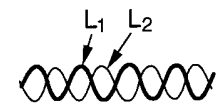

$\phi\left(L_{2}\right)-\phi\left(L_{1}\right)=180^{\circ}$
Fig. 11. Response of the magnetosphere to two types of input time series, a step function (left) and a sinusoid (right), a graphical summary of the numerical study by Itonaga et al. (1997). The wave form and phase relation between two radial distances $L_{1}$ and $L_{2}$ are illustrated at the bottom

field data, they find three cases of 1-2 min oscillations when the satellite was near midnight at radial distances of $12-15 R_{\mathrm{E}}$. The events occurred within a few minutes of substorm onsets. One of these events is reproduced in Fig. 12. The antiphase oscillations in the plasma pressure and magnetic field pressure are explained by a
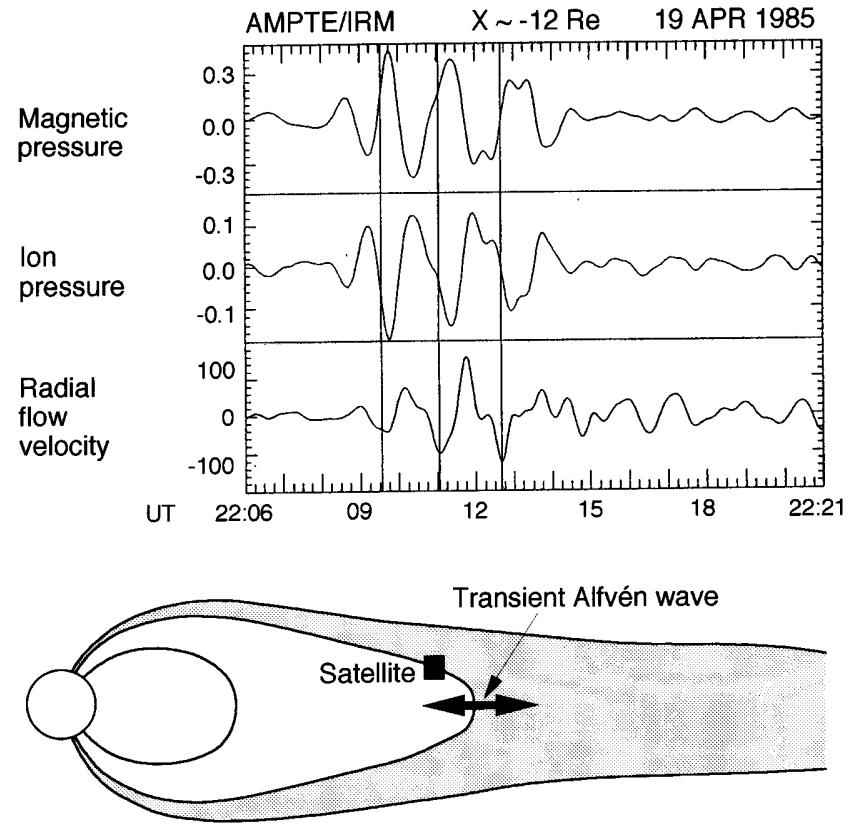

Fig. 12. An example of $\mathrm{Pi}$ 2-band oscillations observed in the magnetotail by the AMPTE/RIM satellite (adapted from Fig. 7 of Bauer et al., 1995). The location of the satellite (solid square) relative to the plasma sheet (shaded region) is schematically illustrated at the bottom radial oscillation of field lines and the associated convection of the pressure gradients. Needless to say, the period of the oscillation is determined by the Alfvén wave bounce time along the relevant field line. Nishida (1979) had proposed that such an oscillation can be excited when the substorm current wedge is formed. Saka et al. (1997) also present a case of simultaneous ion flux modulations and equatorial Pi 2 pulsations and claim that they originate from the same oscillation mode. The test of these Pi 2 models requires more simultaneous observations on the ground and in the magnetosphere. That is, it is necessary to demonstrate, by examining many cases, that the simultaneous observation of particle flux oscillations and Pi 2 pulsations is not a mere coincidence.

\section{Internally excited long-period waves}

Particles in the magnetosphere provide free energy for driving ULF waves. Because the waves generated by the particles tend to azimuthally propagate much more slowly than a fast-mode wave, the azimuthal wave numbers of the waves are large and observation on the ground is sometimes difficult owing to the ionospheric masking effect. Although a basic understanding of the excitation of these waves is obtained from satellite observations and kinetic theories of the instabilities in the ring current (see Takahashi, 1996 for a review) many aspects of the waves still need further investigation.

\subsection{Radial mode structure of drift Alfvén-ballooning waves}

Meridionally polarized magnetic Pc 4-5 pulsations in the magnetosphere are known to be radially localized (e.g., Engebretson et al., 1992), but this observation has not been explained in relation to the excitation mechanism of the pulsations. Vetoulis and Chen (1996) use a kinetic approach to the radial mode structure. In previous theories, an eikonal assumption had been made to render the problem one-dimensional, that is, field-aligned. This is equivalent to assuming an unrealistically small radial extent of the wave field, or the guided poloidal mode of Radoski (1967).

For a magnetosphere with a radially localized plasma, Vetoulis and Chen (1996) find that two regular turning points are produced by a strong earthward pressure gradient that exists at the outer edge of the ring current and that the turning points can localize the unstable drift Alfvén-ballooning modes (DABM). As shown in Fig. 13, the trapping is analogous to the trapping of fast-mode waves by the plasmaspheric turning points, as illustrated in Fig. 7 (top), but for the case of a hot plasma there are complications in deriving the radial mode structure. For example, the trapping becomes more efficient with increasing $m$ (azimuthal mode number), but there is an upper limit to $m$, which is set by the instability mechanism. Also, there is a lower limit to $m$ for the mode to be radially 


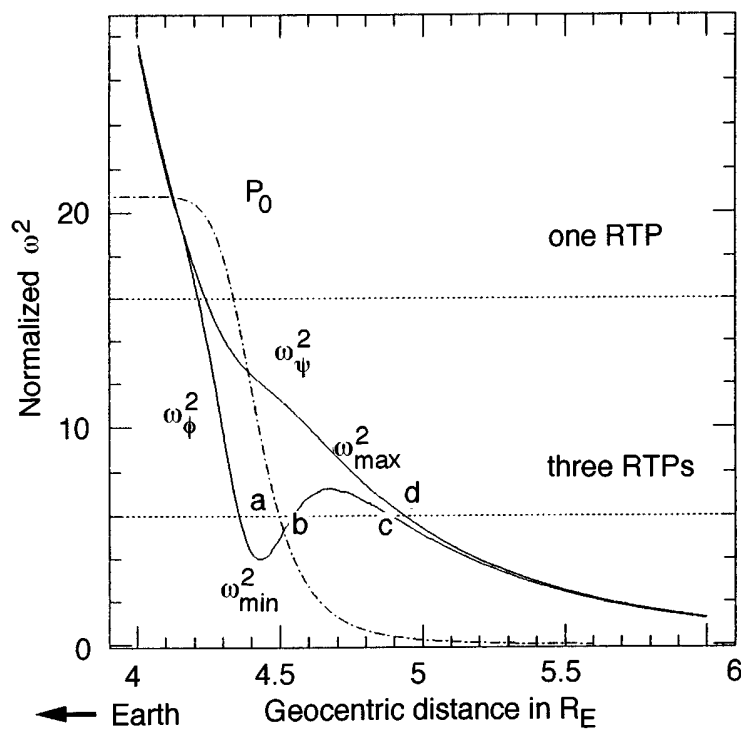

Fig. 13. Radial profiles of the ring current pressure $P_{0}$, and frequencies relevant to the radial confinement of unstable drift Alfvén-ballooning modes (reproduction of Fig. 2 of Vetoulis and Chen, 1996). The dashed curve indicates the pressure. The traces labeled $\omega_{\Psi}^{2}$ and $\omega_{\phi}^{2}$ are local eigenfrequencies associated with radial and azimuthal differential operators. Depending on the frequency (horizontal dotted line) of the global mode, there can be up to three regular turning points (RTPs or cutoff points, $a, b$, and $c$ ). There is always one singular resonant point (or a shear Alfvén resonance at point $d$, for example). The global mode can be trapped between the turning points $a$ and $b$

localized. These results are derived using an analytical method that requires some approximations. A more quantitative description of the spatial mode structure would require a simulation-based approach.

\subsection{Giant pulsations}

When the azimuthal wave number is not very large, the ionospheric masking effect is weak. This means that detectable magnetic field perturbations reach the ground from waves excited within the magnetosphere. Giant pulsations, with a typical azimuthal wave number of 20 , represent such waves, and with multipoint observations on the ground it is possible to determine their radial mode structure without the usual spatial-temporal ambiguity associated with satellite observations. Chisham et al. (1997) construct an average radial structure of giant pulsations using 34 events observed by the EISCAT magnetometer cross, as shown in Fig. 14 (left). The poloidal component ( $\mathrm{D}$ on the ground, assuming a $90^{\circ}$ rotation of polarization axis through the ionosphere) shows a Gaussian latitudinal amplitude profile. In contrast, the toroidal component $(\mathrm{H}$ on the ground) shows a less well-defined profile, with an indication of a plateau or dip at the latitude where the poloidal component reaches a maximum. This result gives convincing support of the radial localization of internally excited pulsations.

In addition, Chisham et al. (1997) demonstrate that it is not necessary to have a detailed wave excitation mechanism to explain the latitudinal amplitude profiles. Using the MHD numerical technique developed by Mann and Wright (1995), Chisham et al. (1997) follow the temporal development of the radially localized transverse large- $m$ pulsations. In the MHD simulation, the initial perturbation is dominated by the poloidal component, for which the latitudinal amplitude variation is modeled by a Gaussian, as shown in Fig. 14 (right). As time progresses, the initially poloidal-dominated wave evolves into a toroidal-dominated wave, and the toroidal profile changes to a single-peaked one from the initial profile that had two peaks. The observed latitudinal amplitude profiles can be explained by the model: the observed poloidal profile is consistent with a single-peak function and the observed toroidal profile is consistent with a superposition of single- and doublepeak profiles that appear at different epochs of wave evolution. It should be noted that the final stage of evolution of the poloidal mode into the toroidal mode (Mann et al., 1997) is not evident in the observed giant pulsations. This could be explained by damping of the wave by ionospheric Joule dissipation, which was not incorporated in the numerical model.

Although the MHD model is successful in explaining the spatial structure of giant pulsations, their excitation mechanism remains a subject of considerable debate. The main question is whether the pulsations are generated by a drift-bounce resonance of energetic magnetospheric ions with antisymmetric field-aligned standing waves, which appears to explain the majority of radially polarized pulsations observed in the magnetosphere (Takahashi and McPherron, 1984), where the symmetry is defined in terms of the filed-line displacement pattern about the magnetic equator. Chisham (1996) favors the antisymmetric mode and offers a model calculation of the drift path of $5-30 \mathrm{keV}$ ions, which might excite the giant pulsations. He shows that the particle can reach the dawn sector only under special circumstances, in agreement with the rare occurrence of giant pulsations. However, he also notes that the particle drift consideration alone does not exclude the possibility of a symmetric mode. In fact, previous satellite observations (e.g., Hillebrand et al., 1982; Takahashi et al., 1992) present evidence against an antisymmetric mode. More observations are needed to determine the standing wave mode and the underlying excitation mechanism of giant pulsations. In particular, it is important to examine the distribution function of energetic particles associated with the wave events. Hillebrand et al. (1982) report modulation of particle fluxes, but it is not clear how the particles are related to the wave-excitation mechanism.

\section{Internally excited short-period waves}

The morphological and theoretical understanding of electromagnetic ion cyclotron waves (Pc 1-2 waves) has greatly improved in recent years owing to extensive analysis of satellite and related ground magnetometer data. Since theoretical topics related to Pc 1-2 waves 

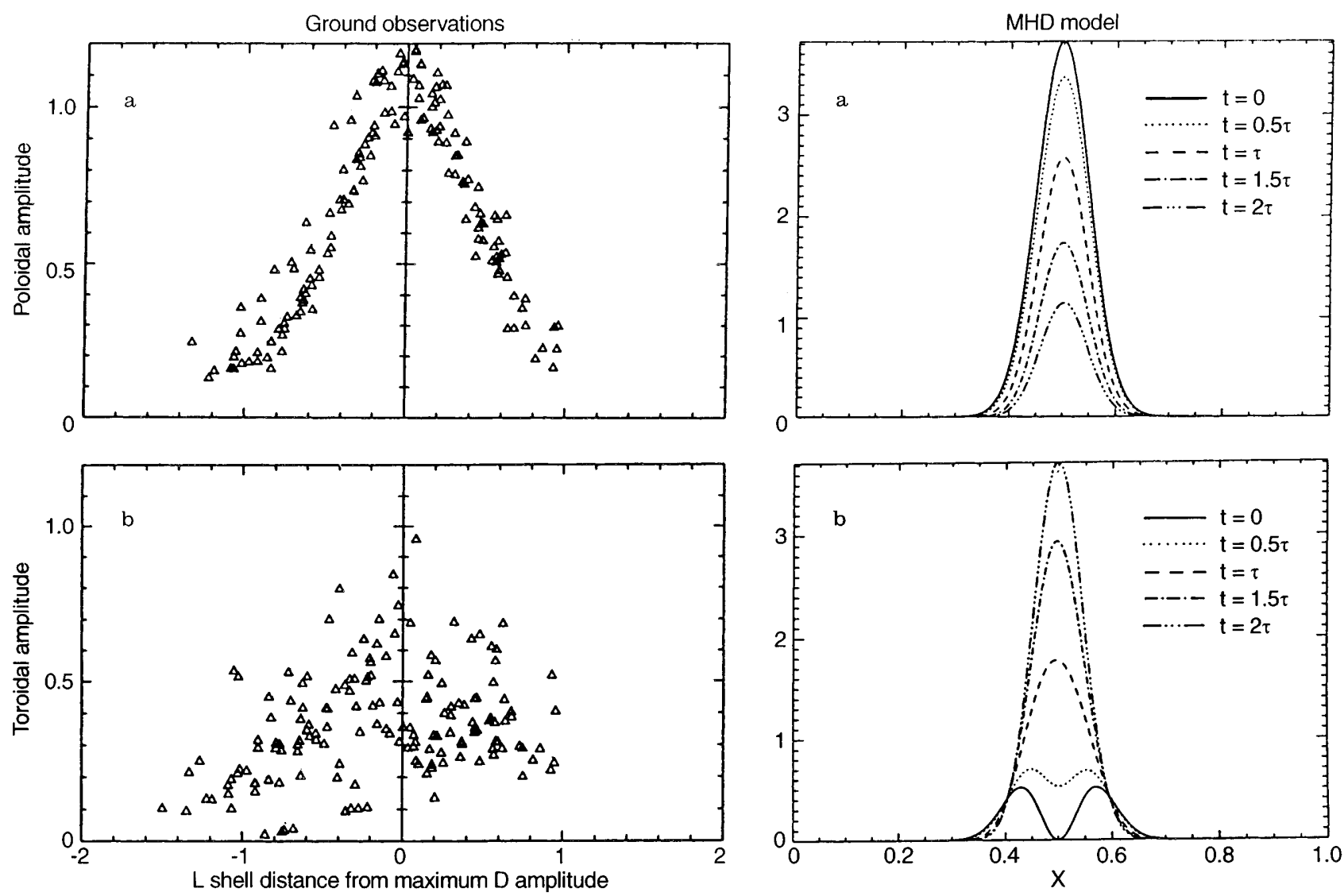

Fig. 14. Comparison of observation (left) and MHD model (right) of the latitudinal amplitude profile of giant pulsations (adapted from Fig. 7 and 8 of Chisham et al., 1997)

were covered in other IAGA division 3 reports, we limit ourselves here to observations of two types of Pc 1-2 waves: Pc 1 bursts in the outer magnetosphere, and structured waves (pearls) in the inner magnetosphere. Also discussed is the quasi-periodic modulation of VLFELF (whistler-mode) waves at Pc 3-4 frequency. This phenomenon is included because it is a high-frequency analogue of Pc 1-2 bursts, yet a different model has been offered regarding the source region.

\subsection{Pc 1 bursts}

There have been suggestions that high-latitude Pc 1 (sometimes extending to Pc 2) bursts originate from the cusp or low-latitude boundary layer (LLBL) (Cole et al. 1982; Morris and Cole, 1985; Hansen et al. 1992). In the model proposed by Cole et al. (1982), the high-latitude Pc 1-2 waves are generated as the hot solar wind ions are mixed with the cold terrestrial ions in the LLBL. The interval of pulsations with rising period (IPRP), typical of the Pc 1 bursts on the ground, is explained by the change in wave frequency as the LLBL source regions is convected tailward.

A number of ground-based experimental studies address Pc 1 source mechanisms and source locations. Neudegg et al. (1995) use a ground magnetometer array in an attempt to determine the source region. Their observations are consistent with a high-latitude source, but the authors are unable to distinguish a source at the cusp, LLBL, and the outer magnetosphere. In contrast, Plyasova-Bakounina et al. (1996), who use an $L=6-9$ magnetometer network, favor a source within the magnetosphere. As noted by Anderson et al. (1996), these different results probably occur from the cutoff effect in the magnetosphere or from the spread of wave signals in the ionospheric wave guide. Convergence of cusp or LLBL field lines to a few degrees of latitude at the ionosphere also makes it difficult to determine the magnetospheric source region. Therefore, satellite observations become crucial for finding the source region.

Anderson et al. (1996) present a CCE satellite-South Pole observation of a sequence of Pc 1-2 bursts and indicate that nearly identical bursts are observed when the satellite is in the outer magnetosphere with its geomagnetic footprint close to South Pole, as illustrated in Fig. 15. On the ground, one of the individual bursts is found to be IPRP, but it was not dispersive at CCE. From this observation it is concluded that the bursts were generated in a region earthward of the LLBL. In addition, using the varying distance between the satellite and ground station, Anderson et al. (1996) estimate that the Pc 1-2 source region is extended 1-2 hours azimuthally and $1-2 R_{\mathrm{E}}$ radially. Satellite data also provide information on the solar wind condition that is related to the triggering of the bursts. At CCE, the 


\section{CCE/South Pole Pc1 Bursts: February 14, 1986}

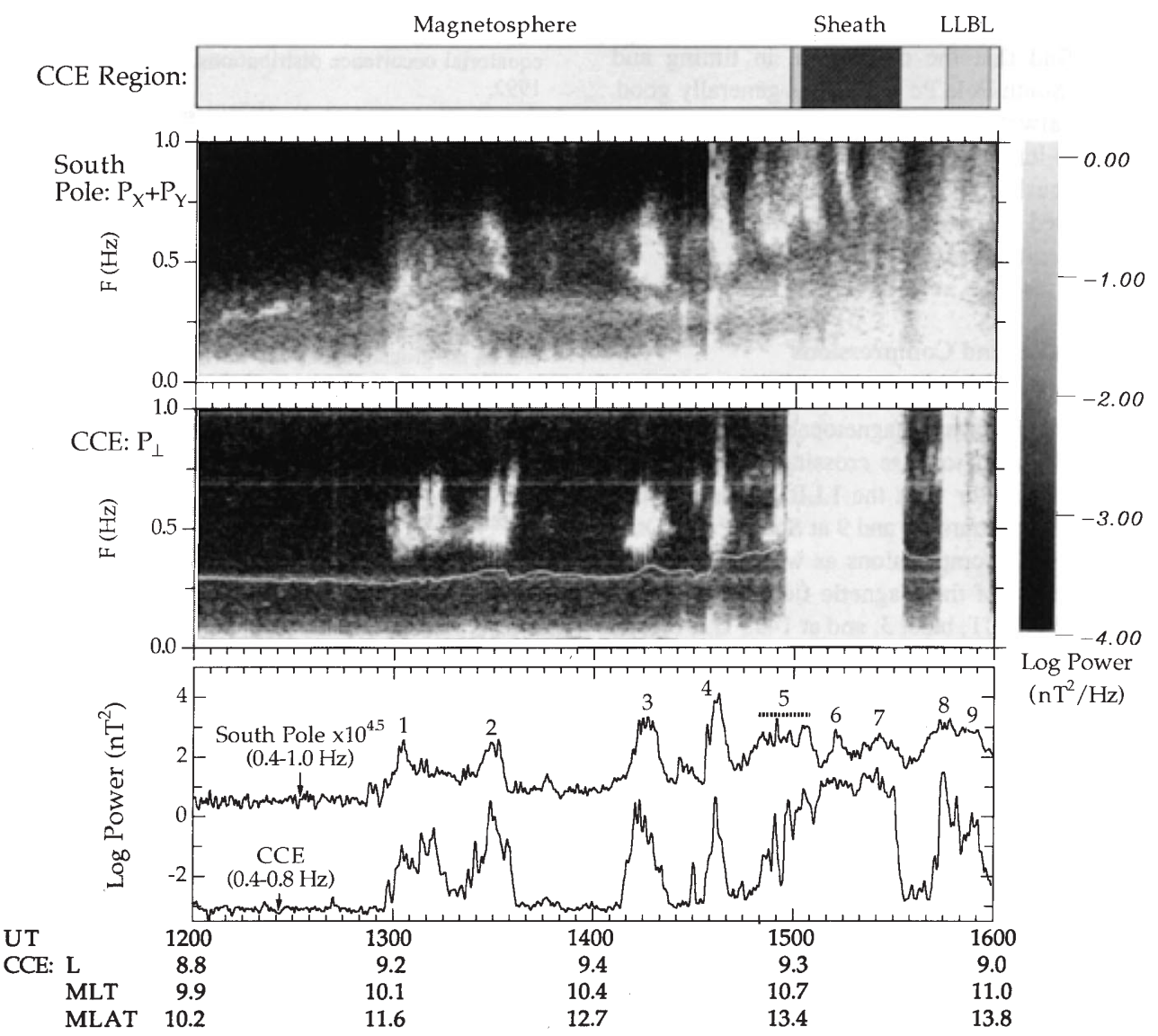

Fig. 15. Simultaneous observations of Pc 1 bursts in the outer magnetosphere by the AMPTE/ CCE satellite and at South Pole. The ground station was located near the geomagnetic field footprint of the satellite (reproduction of Fig. 3 of Anderson et al., 1996) occurrence of the bursts is correlated with the compression of the magnetosphere, presumably by sudden increases in the solar wind pressure.

The correlation between Pc 1 bursts and solar wind pressure changes is supported by Arnoldy et al. (1996), who find simultaneous occurrence of magnetic impulse events (MIEs) and Pc1 bursts at a high rate $(\sim 70 \%)$. For such a magnetospheric source mechanism, the frequency of the waves is expected to rise as the magnetic field compression propagates earthward, i.e., toward the region of stronger magnetic field and hence higher wave frequency. This explains the observation of IPRP on the ground and the absence of dispersion at the satellite: a ground magnetometer samples waves generated over a wide range of latitude, whereas satellite magnetometers sample locally excited waves (Anderson et al. 1996).

\subsection{Structured Pc 1 pulsations}

Another well-known Pc 1 type, the "pearl pulsations" or structured Pc 1 pulsations, are also investigated using ground and satellite observations. In contrast to Pc 1 bursts, structured Pc 1 waves occur deep in the magnetosphere, often inside the plasmasphere. Consequently, the process for modulating the frequency and amplitude of the pulsations must be internal to the magnetosphere. One possible modulation process is via reflection of ion cyclotron wave packets by the northern and southern ionospheres (Jacobs and Watanabe, 1964).

Using CRRES magnetic field and electric field measurements, which provide complete measurements of wave Poynting flux, Fraser et al. (1996) test the concept of a single wave packet bouncing between ionospheres. If this mechanism exists, then the wave energy must propagate past the spacecraft alternatively in opposite directions, and the bouncing packet should be observed twice per "pearl" observed on the ground. Among the 9 Pc 1 events identified in $L=4.8-6.7$, 1300-0100 MLT, and magnetic latitudes between $\pm 20^{\circ}$, none showed the expected signatures of bouncing wave packets. For one example, which exhibited a periodic enhancement in wave power (shown in Fig. 16), the Poynting flux of each of the four wave packets (labeled 1 through 4 in the top trace) was directed away from the equator. Fraser et al. (1996) do not confirm that their events were associated with structured $\mathrm{Pc} 1$ on the ground, so there is some uncertainty in their conclusion. However, the ground-space propagation time delay of a structured Pc 1 event, based on Viking measurement just inside the plasmapause, confirms that structured Pc 1 waves propagate downward (Erlandson et al. 1996).

To summarize, none of the satellite observations supports the presence of Pc 1 wave packets predicted by 
CRRES Orbit 512 February 21, 1991

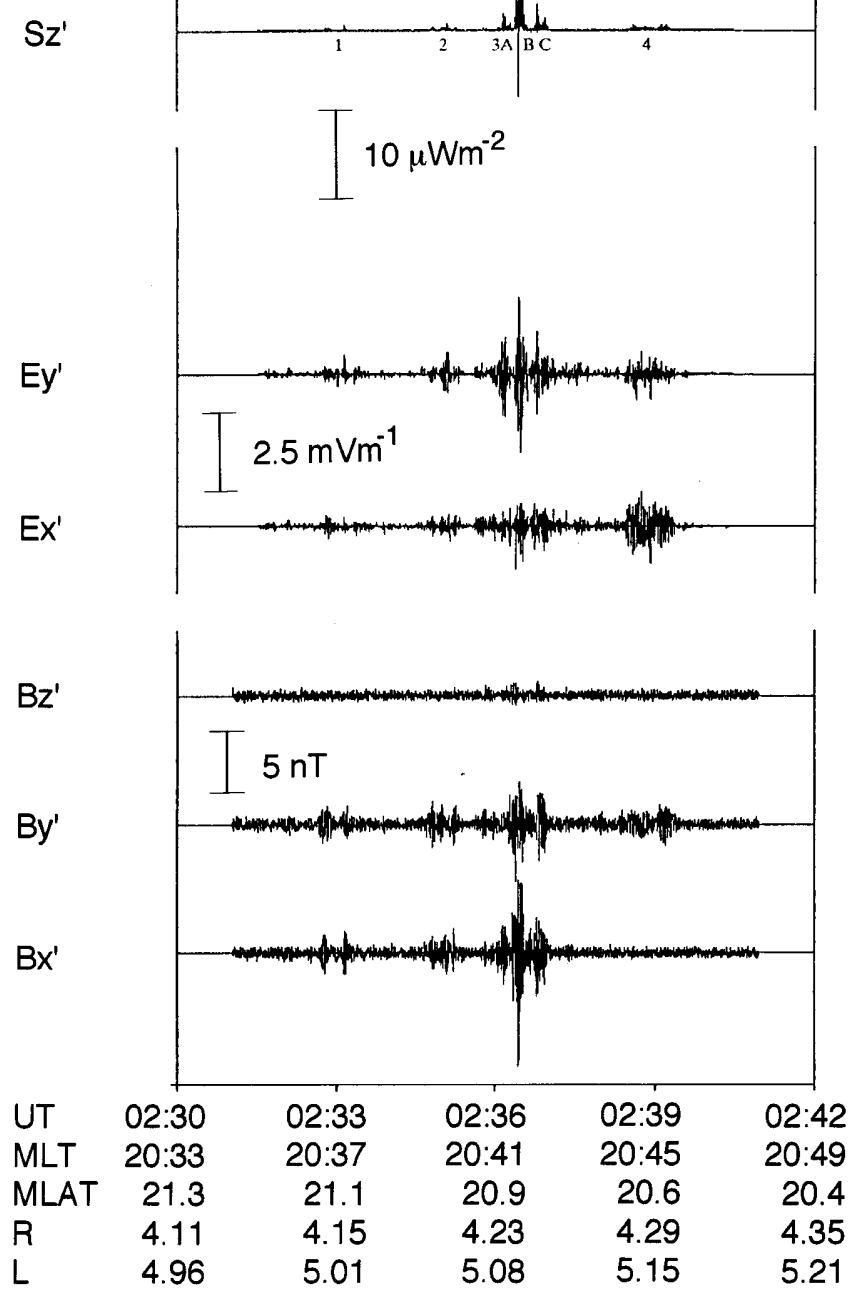

Fig. 16. Poynting flux $\left(S_{z}^{\prime}\right)$, electric field $\left(E_{x}^{\prime}, E_{y}^{\prime}\right)$, and magnetic field $\left(B_{X}^{\prime}, B_{y}^{\prime}, B_{z}^{\prime}\right)$ for a series of Pc 1 waves observed by the CRRES satellite in the plasmasphere (reproduction of Fig. 1 of Fraser et al., 1996). The positive sign of $S_{z}^{\prime}$ means energy propagation away from the equator

the model of a bouncing wave packet. Alternative generation mechanisms, including enormous amplification of a wave packet as its transit through the equator and wave generation by a bounce phase bunched particle population (see Erlandson et al., 1992), have not received conclusive observational support either. The generation mechanism of structured Pc 1 waves remains to be understood.

\subsection{Quasi-periodic ELF-VLF modulation by Pc 3-4 waves}

An electron analogue of Pc 1 bursts is the Type-1 quasiperiodic (QP) amplitude modulation of ELF-VLF waves at Pc 3-4 frequencies. In this case the generation of the ELF-VLF waves is attributed to the wave-particle resonance

$\omega+(\mathbf{k} \cdot \mathbf{V}) \approx \Omega^{-}$

where $\omega$ and $\mathbf{k}$ are the frequency and wave number, $\mathbf{V}$ is the particle (electron) velocity, and $\Omega^{-}$is the electron cyclotron frequency. The resonance condition can be satisfied between whistler waves $\left(\omega<\Omega^{-}\right)$and electrons traveling opposite to each other. The growth of the waves is likely to be stronger when $\Omega^{-}$(or equivalently, the local magnetic field intensity $B$ ) is lower, because the resonance energy will then be lower and more electrons will satisfy the resonance condition. Thus it has been proposed that the equatorial minimum- $B$ region is the source region of the QP emissions. The upstream waves propagating into the equatorial magnetosphere as compressional Pc 3-4 waves are considered to modulate the resonance condition, leading to the QP modulation of the whistler (Sato and Fukunishi, 1981; Morrison and Freeman, 1995).

A statistical study of the probability of occurrence of QP emissions and their dependence on season and the interplanetary magnetic field proposes that the source of the emissions is located in the "horn" region of the magnetosphere, away from the equator (Morrison et al., 1994; Alford et al., 1996). The model by Alford et al. (1996) is illustrated in Fig. 17 and is based on the

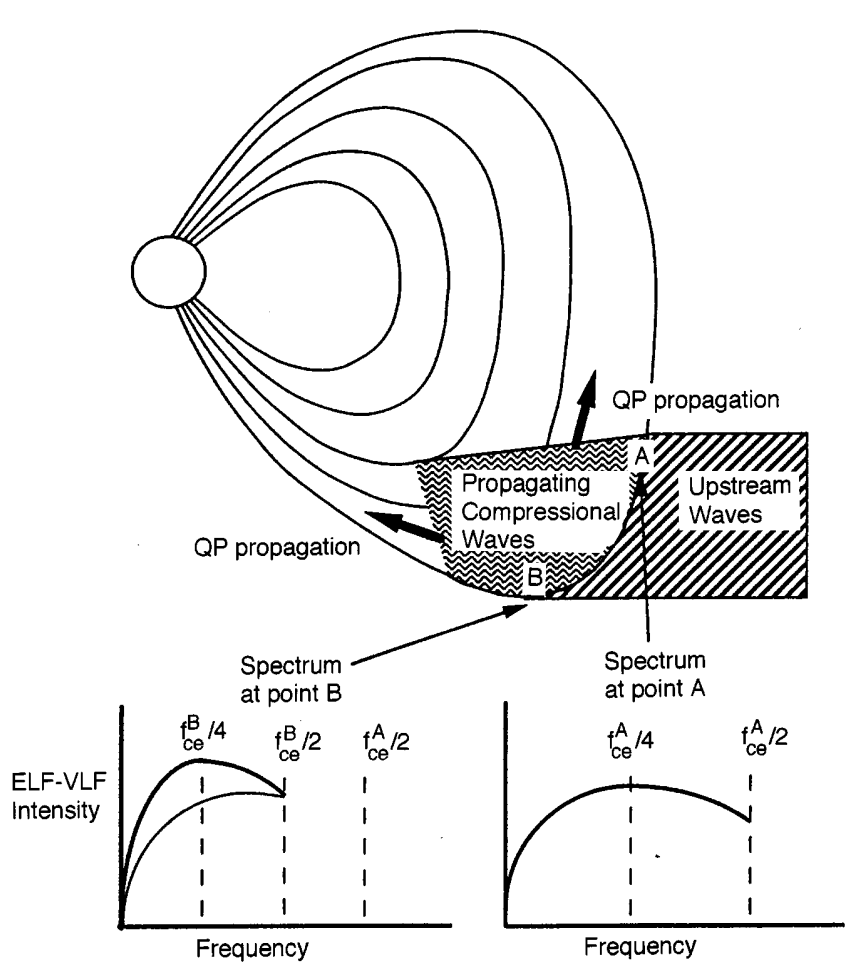

Fig. 17. A model of magnetic field lines and the source region of the Type 1 QP emissions (reproduction of Fig. 11 of Alford et al., 1996). In this schematic version of the Tsyganenko (1991) magnetic field model the field magnitude is minimum in the "horn," where whistler waves are most easily excited. The growth rate of the whistler waves is modulated by Pc 3-4 waves that originate in the upstream region and propagate into the magnetosphere with compressional magnetic field perturbations 
magnetic field configuration given by Tysganenko (1991). The magnetic field in the hatched region (horn) is weaker than in the equatorial region, so the whistler waves are preferentially excited in the horn region. The amplitude of the whistler waves is controlled by the magnetic field compression associated with $\mathrm{Pc}$ 3-4 pulsations transmitted through the high-latitude magnetopause. The magnitude of magnetic field in the horn region changes as the dipole title angle changes. This geometric effect explains why the probability of occurrence of QP emissions in a fixed ELF-VLF band depends on season. Previously, the seasonal variation was linked to the scale height of the ionosphere (Sato et al., 1990). In this model the daily duration of sunlight is the controlling mechanism for the seasonal variation. The two models for the source region can be distinguished if we have satellite measurements at high and equatorial latitudes.

\section{Conclusions}

The general trends in the ULF wave studies are (1) the use of multipoint measurements and statistical studies using a large database, and (2) comparison of observations with numerical models. These trends have resulted in considerable progress over the past few years. Having satellites or ground-based instruments in crucial regions is the key to successful testing of various models of wave excitation and propagation. This trend should continue as data from ISTP and other projects become available.

Acknowledgements. The author is grateful to those who sent reprints while the oral version of this paper was being prepared. Work at the Johns Hopkins University Applied Physics Laboratory was supported by the National Science Foundation and the Office of Naval Research.

\section{References}

Alford, J. L., M. J. Engebretson, R. L. Arnoldy, and U. S. Inan, Frequency variations of quasi-periodic ELF-VLF emissions: a possible new ground-based diagnostic of the outer high-latitude magnetosphere, J. Geophys. Res., 101, 83-97, 1996.

Allan, W., and E. M. Poulter, Damping of magnetospheric cavity modes: a discussion, J. Geophys. Res., 94, 11843$11853,1989$.

Allan, W., and J. R. Manuel, Ponderomotive effects in magnetospheric hydromagnetic waves, (based on 1997 IAGA invited presentation), Ann. Geophysicae., 14, 893-905, 1996.

Allan, W., S. P. White, and E. M. Poulter, Impulse-excited hydromagnetic cavity and field-line resonances in the magnetosphere, Planet. Space Sci., 34, 371-385, 1986a.

Allan, W., E. M. Poulter, and S. P. White, Hydromagnetic wave coupling in the magnetosphere-plasmapause effects on impulseexcited resonances, Planet. Space Sci., 34, 1189-1200, 1986b.

Anderson, B. J., and M. J. Engebretson, Relative intensity of toroidal and compressional Pc 3-4 wave power in the dayside outer magnetosphere, J. Geophys. Res., 100, 95919603, 1995.

Anderson, B. J., M. J. Engebretson, S. P. Rounds, L. J. Zanetti, and T. A. Potemra, A statistical study of Pc 3-5 pulsations observed by the AMPTE/CCE magnetic fields experiment, 1, Occurrence distributions, J. Geophys. Res., 95, 10495-10523, 1990.
Anderson, B. J., R. E. Erlandson, M. J. Engebretson, J. Alford, and R. L. Arnoldy, Source regions of 0.2 to $1.0 \mathrm{~Hz}$ geomagnetic pulsation bursts, Geophys. Res. Lett., 23, 769-772, 1996.

Arnoldy, R. L., M. J. Engebretson, J. L. Alford, R. E. Erlandson, and B. J. Anderson, Magnetic impulse events and associated Pc 1 bursts at dayside high latitudes, J. Geophys. Res., 101, 77937799, 1996.

Bauer, T. M., W. Baumjohann, and R. A. Treumann, Neutral sheet oscillations at substorm onset, J. Geophys. Res., 100, $23737-$ 23742, 1995.

Bellan, P. M., Alfvén resonance reconsidered: exact equations for wave propagation across a cold inhomogeneous plasma, Phys. Plasmas, 1, 3523-3541, 1994.

Bellan, P. M., Mode conversion into non-MHD waves at the Alfvén layer: the case against the field line resonance concept, J. Geophys. Res., 101, 24887-24898, 1996.

Björnsson, A., Q. Hillebrand, and H. Voelker, First observational results of geomagnetic Pi 2 and Pc 5 pulsations on a north-south profile through Europe, Z. Geophys., 37, 1031-1042, 1971.

Bradshaw, E. G., and M. Lester, SABRE observations of Pi 2 pulsations: case studies, Ann. Geophysicae., 15, 40-53, 1997.

Chisham, G., Giant pulsations: an explanation for their rarity and occurrence during geomagnetically quiet times, J. Geophys. Res., 101, 24755-24763, 1996.

Chisham, G., I. R. Mann, and D. Orr, A statistical study of giant pulsation latitudinal polarization and amplitude variation, J. Geophys. Res., 102, 9619-9629, 1997.

Cole, K., R. J. Morris, E. T. Matveyeva, V. A. Troitskaya, and O. A. Pokhotelov, The relationship of the boundary layer of the magnetosphere to IPRP events, Planet. Space Sci., 30, 129-136, 1982.

Engebretson, M. J., L. J. Zanetti, T. A. Potemra, and M. H. Acuna, Harmonically structured ULF pulsations observed by the AMPTE CCE magnetic field experiment, Geophys. Res. Lett., 13, 905-908, 1986.

Engebretson, M. J., Catching the wave: ULF research in the U.S. since 1991, US National Report to IUGG, 1991-1994, Rev. Geophys. 33, Suppl., 693-701, 1995.

Engebretson, M. J., D. L. Murr, K. N. Erickson, R. J. Strangeway, D. M. Klumpar, S. A. Fuselier, L. J. Zanetti, and T. A. Potemra, The spatial extent of radial magnetic pulsation events observed in the dayside near synchronous orbit, J. Geophys. Res., 97, 13741-13758, 1992.

Erlandson, R. E., B. J. Anderson, L. J. Zanetti, and L. P. Block, Viking satellite observations of periodic Pc 1 waves: pearl pulsations, J. Geophys. Res., 97, 14823-14832, 1992.

Erlandson, R. E., K. Mursula, and T. Bosinger, Simultaneous ground-satellite observations of structured Pc 1 pulsations, J. Geophys. Res., 101, 27149-27156, 1996.

Feng, Q., B. J. Fraser, F. W. Menk, C. W. S. Ziesolleck, O. Saka, and T. Kitamura, Pc 3-4 geomagnetic pulsation observed at very low latitude conjugate stations, J. Geophys. Res., 100, 1928719298, 1995.

Feygin, F. Z., O. A. Pokhotelov, D. O. Pokhotelov, T. Bräysy, J. Kangas, and K. Mursula, Exo-plasmaspheric refilling due to ponderomotive forces induced by geomagnetic pulsations, J. Geophys. Res., 102, 4841-4845, 1997.

Fraser, B. J., H. J. Singer, W. J. Hughes, J. R. Wygant, R. R. Anderson, and Y. D. Hu, CRRES Poynting vector observations of electromagnetic ion cyclotron waves near the plasmapause, J. Geophys. Res., 101, 15331-15343, 1996.

Francia, P., and U. Villante, Some evidence of ground power enhancements at frequencies of global magnetospheric modes at low latitudes, Ann. Geophysicae, 15, 12-23, 1997.

Fujita, S., and K. -H. Glassmeier, Magnetospheric cavity resonance oscillations with energy flow across the magnetopause, J. Geomag. Geoelectr., 47, 1277-1292, 1995.

Guglielmi, A. V., O. A. Pokhotelov, F. Z. Feygin, Yu. P. Kurchashov, J. F. McKenzie, P. K. Shukla, L. Stenflo, and A. S. Potapov, Ponderomotive wave forces in longitudinal MHD waveguides, J. Geophys. Res., 100, 7997-8002, 1995. 
Hansen, H. J., B. J. Fraser, F. W. Menk, Y.-D. Hu, P. T. Newell, C. -I. Meng, and R. J. Morris, High-latitude Pc 1 bursts arising in the dayside boundary layer region, J. Geophys. Res., 97, 39934008, 1992.

Harrold, B. G., and J. C. Samson, Standing ULF modes of the magnetosphere: a theory, Geophys. Res. Lett., 19, 1811-1814, 1992.

Hillebrand, O., J. Munch, and R. L. McPherron, Ground-satellite correlative study of a giant pulsation event, J. Geophys. Res., 51, 129-140, 1982.

Itonaga, M., A. Yoshikawa, and K. Yumoto, One-dimensional transient response of the inner magnetosphere at the magnetic equator. 2. Analysis of waveforms, J. Geomag. Geoelectr., 49, 49-68, 1997.

Jacobs, J. A., and T. Watanabe, Micropulsations whistlers, J. Atmos. Terr. Phys., 26, 825-829, 1964.

Kivelson, M. G., and D. J. Southwood, Coupling of global magnetospheric MHD eigenmodes to field line resonances, J. Geophys. Res., 91, 4345-4351, 1986.

Kivelson, M. G., J. Etcheto, J. G. Trotignon, Global compressional oscillations of the terrestrial magnetosphere: the evidence and a model, J. Geophys. Res., 89, 9851-9856, 1984.

Korotova, G. I., and D. G. Sibeck, A case study of transient event motion in the magnetosphere and in the ionosphere, J. Geophys. Res., 100, 35-46, 1995.

Lee, D.-H., Dynamics of MHD wave propagation in the lowlatitude magnetosphere, J. Geophys. Res., 101, 15371-15386, 1996.

Lee, D.-H., and R. L. Lysak, Magnetospheric ULF wave coupling in the dipole field: the impulsive excitation, J. Geophys. Res., 94, 17097-17103, 1989.

Lee, D.-H., and R. L. Lysak, Impulsive excitation of ULF waves in the three-dimensional dipole model: the initial results, J. Geophys. Res., 96, 3479-3486, 1991a.

Lee, D.-H., and R. L. Lysak, Monochromatic ULF wave excitation in the dipole magnetosphere, J. Geophys. Res., 96, 5811-5817, $1991 b$.

Lin, N., M. J. Engebretson, L. A. Reinleitner, J. V. Olson, D. L. Gallagher, L. H. Cahill, Jr., J. A. Slavin, and A. M. Persoon, Field and thermal plasma observations of ULF pulsations during a magnetically disturbed interval, J. Geophys. Res., 97, 14859-14875, 1992.

Mann, I. R., and A. N. Wright, Finite lifetimes of ideal poloidal Alfven waves, J. Geophys. Res., 100, 23677-23686, 1995.

Mann, I. R., A. N. Wright, and A. W. Hood, Multiple-time scales analysis of ideal poloidal Alfvén waves, J. Geophys. Res., 102, 2381-2390, 1997.

Matsuoka, H., K. Takahashi, K. Yumoto, B. J. Anderson, and D. G. Sibeck, Observation and modeling of compressional Pi 3 magnetic pulsations, J. Geophys. Res., 100, 1210312115, 1995.

Matsuoka, H., K. Takahashi, S. Kokubun, K. Yumoto, T. Yamamoto, S. I. Solovyev, and E. F. Vershinin, Phase and amplitude structure of Pc 3 magnetic pulsations as determined from multipoint observations, J. Geophys. Res., 102, 2391-2403, 1997.

Morris, R. J., and K. D. Cole, Pc 1-2 discrete regular daytime pulsation bursts at high latitudes, Planet. Space Sci., 33, 53-67, 1985.

Morrison, K., and M. P. Freeman, The role of ULF waves in the generation of quasi-periodic ELF-VLF emissions, Ann. Geophysicae, 13, 1127-1133, 1995.

Morrison, K., M. J. Engebretson, J. R. Beck, J. E. Johnson, R. L. Arnoldy, L. J. Cahill Jr., D. L. Carpenter, and M. Gallani, A study of quasi-periodic ELF-VLF emissions at three Antarctic stations: Evidence for off-equatorial generation?, Ann. Geophysicae, 12, 139-146, 1994

Neudegg, D. A., B. J. Fraser, F. W. Menk, H. J. Hansen, G. B. Burns, R. J. Morris, and M. J. Underwood, Sources and velocities of Pc 1-2 ULF waves at high laitudes, J. Geophys. Res., 22, 2956-2968, 1995.
Nishida, A., Possible origin of transient dusk-to-dawn electric field in the nightside magnetosphere, J. Geophys. Res., 84, 34093412, 1979.

Nosé, M., T. Iyemori, M. Sugiura, and J. A. Slavin, A strong dawn/ dusk asymmetry in Pc 5 pulsation occurrence observed by the DE-1 satellite, Geophys. Res. Lett., 22, 2053-2056, 1995.

Plyasova-Bakounina, T. A., J. Kangas, K. Mursula, O. A. Molchanov, and A. W. Green, Pc 1-2 and Pc 4-5 pulsations observed at a network of high-latitude stations, J. Geophys. Res., 101, 1096510973, 1996.

Potemra, T. A., and L. G. Blomberg, A survey of Pc 5 pulsations in the dayside high-latitude regions observed by Viking, J. Geophys. Res., 101, 24801-24813, 1996.

Provan G., and T. K. Yeoman, A comparison of field-line resonances observed at the Goose Bay and Wick radars, Ann. Geophysicae, 15, 231-235, 1997.

Radoski, H. R., Highly asymmetric MHD resonances: the guided poloidal mode, J. Geophys. Res., 72, 4026-4027, 1967.

Rankin, R., P. Frycz, V. T. Tikhonchuk, and J. C. Samson, Ponderomotive saturation of magnetospheric field line resonances, Geophys. Res. Lett., 22, 1741-1744, 1995.

Rickard, G. J., and A. N. Wright, ULF pulsations in a magnetospheric waveguide: comparison of real and simulated satellite data, J. Geophys. Res., 100, 3531-3537, 1995.

Saito, T., and S. Matsushita, Solar cycle effects on geomagnetic Pi 2 pulsations, J. Geophys. Res., 73, 267-286, 1968.

Saka, O., K. Okada, O. Watanabe, D. N. Baker, G. D. Reeves, and R. D. Belian, Pi 2-associated particle flux and magnetic field modulations in geosynchronous altitude, J. Geophys. Res., 102, 11363-11373, 1997.

Samson, J. C., R. A. Greenwald, J. M. Ruohoniemi, T. J. Hughes, and D. D. Wallis, Magnetometer and radar observations of magnetohydrodynamic cavity modes in the earth's magnetosphere, Can. J. Phys., 69, 929-937, 1991.

Samson, J. C., B. G. Harrold, J. M. Ruohoniem, R. A. Greenwald, and A. D. M. Walker, Field line resonances associated with MHD waveguides in the magnetosphere, Geophys. Res. Lett., 19, 441-444, 1992.

Samson, J. C., C. L. Waters, F. W. Menk, and B. J. Fraser, Fine structure in the spectra of low latitude field line resonances, Geophys. Res. Lett., 22, 2111-2114, 1995.

Sarafopoulos, D. V., Long duration Pc 5 compressional pulsations inside the Earth's magnetotail lobes, Ann. Geophysicae, 13, 926937, 1995.

Sato, N., H. Suzuki, and T. Saemundsson, Conjugacy of daytime ELF-VLF emission activities in the auroral zones, J. Geophys. Res., 95, 7847-7856, 1990.

Sato, N., and H. Fukunishi, Interaction between ELF-VLF emissions and magnetic pulsations: classification of quasiperiodic ELF-VLF emissions based on frequency-time spectra, J. Geophys. Res., 86, 19-29, 1981.

Shimazu, H., T. Araki, T. Kamei, and H. Hanado, A symmetric appearance of Pc 5 on dawn and dusk side associated with solar wind dynamic pressure enhancement, J. Geomag. Geoelectr., 47, 177-189, 1995.

Sibeck, D. G., R. E. Lopez, and E. C. Roelof, Solar wind control of the magnetopause shape, location, and motion, J. Geophys. Res., 96, 5489-5495, 1991.

Southwood, D. J., Some features of field line resonances in the magnetosphere, Planet. Space Sci., 22, 483-491, 1974.

Takahashi, K., New observations, new theoretical results and controversies regarding Pc 3-5 waves, Adv. Space Res., 17(10), 63-71, 1996.

Takahashi, K., and R. L. McPherron, Standing hydromagnetic oscillations in the magnetosphere, Planet. Space Sci., 32, 13431359, 1984.

Takahashi, K., N. Sato, J. Warnecke, H. Lühr, H. E. Spence, and Y. Tonegawa, On the standing wave mode of giant pulsations, J. Geophys. Res., 97, 10717-10732, 1992.

Takahashi, K., S. Ohtani, and B. J. Anderson, Statistical analysis of Pi 2 pulsations observed by the AMPTE CCE spacecraft in 
the inner magnetosphere, J. Geophys. Res., 100, 21929-21941, 1995.

Troitskaya, V. A., T. A. Plyasova-Bakounina, and A. V. Gul'elmi, Relationship between Pc 2-4 pulsations and the interplanetary field, Dokl. Akad. Nauk USSR, 197, 1312, 1971.

Uberoi, C., Alfvén waves in the magnetospheric plasma: micropulsations and particle acceleration, Phys. Scripta, T60, 20-31, 1995.

Tsyganenko, N. A., Methods for quantitative modeling of the magnetic field from Birkeland currents, Planet. Space Sci., 39, 641-654, 1991.

Vellante, M., U. Villante, M. De Lauretis, and G. Barchi, Solar cycle variation of the dominant frequencies of Pc 3 geomagnetic pulsations at $\mathrm{L}=1.6$, Geophys. Res. Lett., 23, 1505-1508, 1996.

Vetoulis, G. and L. Chen, Kinetic theory of geomagnetic pulsations 3. Global analysis of drift Alfvén-ballooning modes, J. Geophys. Res., 101, 15441-15456, 1996.

Walker, A. D. M., J. M. Ruohouniemi, K. B. Baker, R. A. Greenwald, and J. C. Samson, Spatial and temporal behavior of ULF pulsations observed by the Goose Bay HF radar, J. Geophys. Res., 97, 12187-12202, 1992.
Waters, C. L., J. C. Samson, and E. F. Donovan, The temporal variation of the frequency of high latitude field line resonances, J. Geophys. Res., 100, 7987-7996, 1995.

Wright, A. N. and W. Allan, Are two-fluid effects relevant to ULF pulsations?, J. Geophys. Res., 101, 24991-24996, 1996a.

Wright, A. N. and W. Allan, Structure, phase motion, and heating within Alfven resonances, J. Geophys. Res., 101, 17399-17408, 1996b.

Yeoman, T. K., and D. Orr, Phase and spectral power of midlatitude Pi 2 pulsations: evidence for a plasmaspheric cavity resonance, Planet. Space Sci., 37, 1367-1383, 1989.

Yumoto, K., Generation and propagation mechanisms of lowlatitude magnetic pulsations - a review, J. Geophys., 60, 79-105, 1986.

Zhu, X. M., and M. G. Kivelson, Global mode ULF pulsations in a magnetosphere with a nonmonotonic Alfvén velocity profile, J. Geophys. Res., 94, 1479-1485, 1989.

Ziesolleck, C. W. S., and D. R. McDiarmid, Statistical survey of auroral latitude Pc 5 spectral and polarization characteristics, J. Geophys. Res., 100, 19299-19312, 1995. 